\title{
GRB 060605: multi-wavelength analysis of the first GRB observed using integral field spectroscopy ${ }^{\star}$
}

\author{
P. Ferrero ${ }^{1}$, S. Klose ${ }^{1}$, D. A. Kann ${ }^{1}$, S. Savaglio ${ }^{2}$, S. Schulze ${ }^{1}$, E. Palazzi ${ }^{3}$, E. Maiorano ${ }^{3,4}$, P. Böhm ${ }^{5}$, D. Grupe ${ }^{6}$, \\ S. R. Oates ${ }^{7}$, S. F. Sánchez ${ }^{8}$, L. Amati ${ }^{3}$, J. Greiner ${ }^{2}$, J. Hjorth ${ }^{9}$, D. Malesani ${ }^{9}$, S. D. Barthelmy ${ }^{10}$, J. Gorosabel ${ }^{11}$, \\ N. Masetti ${ }^{3}$, and M. M. Roth ${ }^{5}$
}

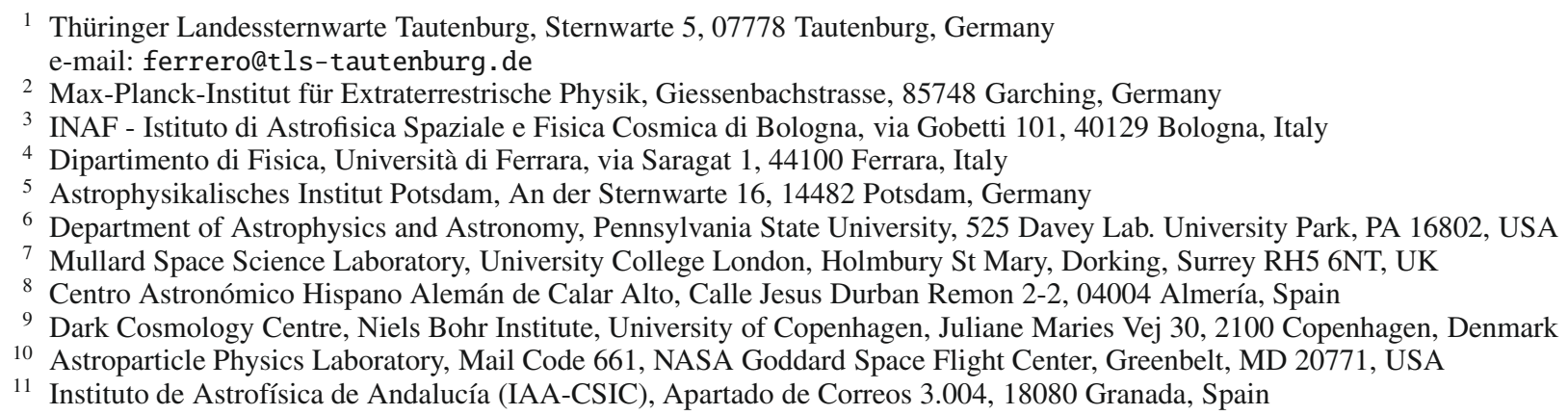

Received 15 April 2008 / Accepted 12 February 2009

\section{ABSTRACT}

\begin{abstract}
The long and relatively faint gamma-ray burst GRB 060605 detected by Swift/BAT lasted about $20 \mathrm{~s}$. Its afterglow could be observed with Swift/XRT for nearly 1 day, while Swift/UVOT could detect the afterglow during the first $6 \mathrm{~h}$ after the event. Here, we report on integral field spectroscopy of its afterglow performed with PMAS/PPak mounted at the Calar Alto $3.5 \mathrm{~m}$ telescope. In addition, we report on a detailed analysis of XRT and UVOT data and on the results of deep late-time VLT observations that reveal the GRB host galaxy. We find that the burst occurred at a redshift of $z=3.773$, possibly associated with a faint, $R_{\mathrm{C}}=26.4 \pm 0.3$ host. Based on the optical and X-ray data, we deduce information on the SED of the afterglow, the position of the cooling frequency in the SED, the nature of the circumburst environment, its collimation factor, and its energetics. We find that the GRB fireball was expanding into a constant-density medium and that the explosion was collimated with a narrow half-opening angle of about 2.4 degrees. The initial Lorentz factor of the fireball was about 250; however, its beaming-corrected energy release in the gamma-ray band was comparably low. The optical, X-ray afterglow, on the other hand, was rather luminous. Finally, we find that the data are consistent within the error bars with an achromatic evolution of the afterglow during the suspected jet break time at about 0.27 days after the burst.
\end{abstract}

Key words. gamma rays: bursts

\section{Introduction}

Since its launch in November 2004, the Swift satellite (Gehrels et al. 2004) has localised more than 300 gamma-ray bursts (GRBs) with an accuracy of 3 to 4 arcmin radius for the satellitebased analysis and 1 arcmin for the ground-based one, using the BAT detector (Barthelmy et al. 2005). Of these, $\sim 84 \%$ could be localised with the Swift X-ray telescope (XRT; Burrows et al. 2005) and $\sim 72 \%$ had an optical/near-infrared afterglow. For $\sim 30 \%$ of the entire sample, it was possible to measure a redshift (see also Greiner's internet page at http: //www . mpe . mpg. de/ jcg/grbgen.html).

The most widely accepted GRB model is the fireball model (e.g., Cavallo \& Rees 1978; Rees \& Mészáros 1992; Sari et al. 1998; for reviews: Piran 2004; Mészáros 2006). Within its framework the burst is accompanied by a relativistic, collimated

\footnotetext{
* Based on observations collected at the German-Spanish Calar Alto Observatory in Spain (Programme F06-3.5-055) and at the European Southern Observatory, La Silla and Paranal, Chile (ESO Programme 177.D-0591).
}

outflow that sweeps up the surrounding interstellar medium. The shocks that form convert the kinetic energy of the flow into internal energy of accelerated particles, which in turn emit synchrotron radiation from X-ray to radio wavelengths. The afterglows are usually identified as either new objects in comparison to archival images or by their fading behaviour. The study and detection of the afterglows enable sub-arcsecond localisation of the burst and unambigous determination of its host galaxy and its redshift if the afterglow is bright enough. The afterglow itself provides information about the physical processes that work and can reveal clues to the nature of the central engine and to the environmental properties of the progenitors. Most of these data can only be derived via a spectroscopic analysis of the optical and X-ray afterglow.

Even though optical afterglows can be very bright at the beginning, the rapid fading of these transients makes the timing of observations crucial for the acquisition of spectroscopic data with a sufficient signal-to-noise $(\mathrm{S} / \mathrm{N})$ ratio. However, due to the time usually needed to identify the optical transient in a GRB X-ray error circle, rapid spectroscopic follow-up 
observations are a challenge. Indeed long-slit spectroscopy has to await the identification of the afterglow, or a best guess has to be made; i.e., if the error box is very small, one can assume that the afterglow is the brightest object in the field. Integral field spectroscopy (IFS), on the other hand, using integral field units (IFUs), can start getting spectra of an entire error box as soon as an arcsecond X-ray location has been reported, usually in the case of the Swift satellite within minutes after the GRB trigger. In principle, once the afterglow has been identified by other means, IFS data could then be used to extract early spectra. This procedure would minimise an important bias, namely the pre-selection of afterglows for spectroscopic follow-up observations according to their apparent magnitude at the time of their discovery. Furthermore, in the Swift era, many optical afterglows are discovered first by the Swift UV/optical telescope which has only filters up to the $v$ band precluding the rapid localisation of $z \gtrsim 5$ or of highly extinguished afterglows (cf. Roming et al. 2006). Needing only Swift XRT localisations, IFS is basically not affected by this colour-selection bias.

Motivated by the aforementioned potential advantages of IFS, we have started an observing campaign of GRB afterglows with IFUs. Here, we report on our first successful IFS observations of an afterglow (GRB 060605) performed at the Calar Alto $3.5 \mathrm{~m}$ telescope. In addition, we report on the analysis of the Swift BAT, XRT and UVOT data and late-time VLT observations.

Throughout this paper we adopt a world model with $H_{0}=$ $71 \mathrm{~km} \mathrm{~s}^{-1} \mathrm{Mpc}^{-1}, \Omega_{\mathrm{M}}=0.27, \Omega_{\Lambda}=0.73$ (Spergel et al. 2003). For the flux density of the afterglow we use the usual convention $F_{v}(t) \propto t^{-\alpha} v^{-\beta}$.

\section{Observations and data reduction}

\subsection{Swift BAT data: the burst}

GRB 060605 was detected by the BAT instrument on-board Swift on June 5, at $T_{0}=18: 15: 44.61$ UT (trigger 213630; Page et al. 2006) with an accuracy of 3 arcmin radius (90\% containment, including systematic uncertainty). The BAT on-board calculated location of the burst was RA $(\mathrm{J} 2000)=21^{\mathrm{h}} 28^{\mathrm{m}} 35^{\mathrm{s}}$ and Dec $=-06^{\circ} 3^{\prime} 36^{\prime \prime}$ (Page et al. 2006), while ground analysis resulted in coordinates RA $(\mathrm{J} 2000)=21^{\mathrm{h}} 28^{\mathrm{m}} 37.6^{\mathrm{s}}$ and Dec $=$ $-06^{\circ} 2^{\prime} 44^{\prime \prime} .7$ with an accuracy of 1.5 arcmin radius.

The time-averaged spectrum of the burst (from $T_{0}-2.580 \mathrm{~s}$ to $T_{0}+20.450 \mathrm{~s}$ ) can be described by a cutoff power law with $\alpha=0.3_{-0.9}^{+0.7}$, and the peak energy at $90_{-20}^{+150} \mathrm{keV}$ (Butler et al. 2007). According to Sato et al. (2006), in the $15-350 \mathrm{keV}$ band the burst had a duration of $T_{90}=15 \pm 2 \mathrm{~s}$, while according to Butler et al. (2007) $T_{90}=19 \pm 1 \mathrm{~s}$.

\subsection{Swift $X R T$ data}

X-ray data of the afterglow of GRB 060605 were collected on 5 and 6 June 2006 with XRT. Pointed observations on target started $93 \mathrm{~s}$ after the BAT trigger and the monitoring was organized in two sequences, with a total net exposure time of $\sim 37.4 \mathrm{ks}$ in photon counting (PC) mode and $\sim 13 \mathrm{~s}$ in windowed timing (WT) mode. In order to obtain a better $\mathrm{S} / \mathrm{N}$ ratio in the spectral analysis, only the data of the first $\sim 30.5 \mathrm{ks}$ of the PC mode observation were used.

The data reduction was performed using the XRTDAS v2.0.1 standard data pipeline package (xrtpipeline v0.10.3), in order to produce the final cleaned event files.

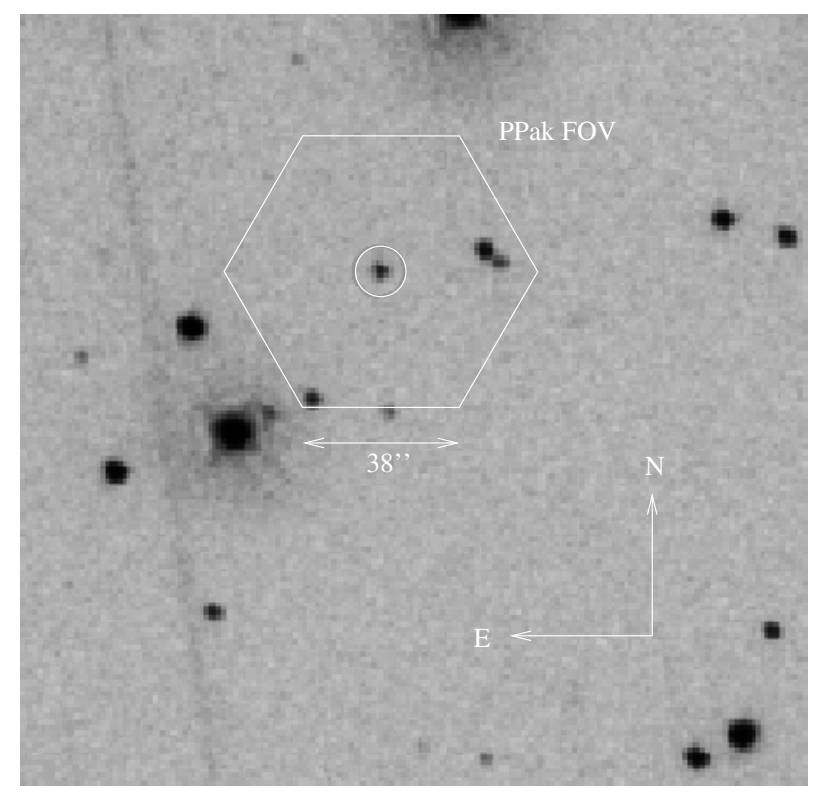

Fig. 1. Swift UVOT $v$-band image of the field of GRB 060605. The optical afterglow is indicated by a circle. The overplotted hexagon shows the sky coverage of PMAS/PPak during our observing campaign (see also Fig. 2).

During sequence 000 the count rate of the burst was high enough to cause pile-up in the PC mode data, which covered the entire first three orbits of XRT observation from $T_{0}+126 \mathrm{~s}$ to about $T_{0}+1.8 \times 10^{4} \mathrm{~s}$. Therefore, to account for this effect, the PC data were extracted in a circle of 25 pixels radius, with a circular region of 4 pixels radius excluded from its centre. The size of the inner region was determined following the procedure described in Vaughan et al. (2006).

The X-ray background was measured within a circle with 40 pixels radius located far from any source. The ancillary response file was generated with the task xrtmkarf (v0.5.2) within FTOOLS ${ }^{1}$ (Blackburn 1995), and accounts for the size of the extraction region. We used the latest spectral redistribution matrices (swxpc0to12_20010101v008.rmf) in the Calibration Database $^{2}$ (CALDB 2.3) maintained by HEASARC.

\subsection{Swift UVOT data}

Swift started settled observations of GRB 060605 with its UV/Optical Telescope (Roming et al. 2005) $78 \mathrm{~s}$ after the trigger. The very first image was in the $v$-band, while the satellite was slewing. Swift found an afterglow at coordinates RA (J2000) = $21^{\mathrm{h}} 28^{\mathrm{m}} 37.32$ and Dec $=-06^{\circ} 3^{\prime} 31^{\prime \prime} .3$ (Page et al. 2006), confirming the optical transient already identified at that time by the robotic ROTSE IIIa telescope (Rykoff et al. 2006).

The afterglow was only detected in the white, $v$ (see Fig. 1), and $b$ filters. The lack of detection in the $U V$ filters (Blustin \& Page 2006) is consistent with the redshift of $z=3.7-3.8$ based on observations with the Australian National University ANU 2.3-m (Peterson \& Schmidt 2006) and the 10-m Southern African Large Telescope (SALT; Still et al. 2006).

The initial observations, namely the white and $v$ finding charts, were performed in event mode (photon counting), while

\footnotetext{
1 http://heasarc.gsfc.nasa.gov/ftools/

2 http://heasarc.gsfc.nasa.gov/docs/heasarc/caldb/ caldb_intro.html
} 
the rest of the exposures were taken predominately in image mode.

The source counts were extracted using a region of $5^{\prime \prime}$ radius. As the source fades it is more accurate to use smaller source apertures (Poole et al. 2008). Therefore, when the count rate fell below 0.5 counts $\mathrm{s}^{-1}$, the source counts were extracted using a region with $3^{\prime \prime}$ radius. These counts were corrected to $5^{\prime \prime}$ using the curve of growth contained in the calibration files. Background counts were extracted using a circular region of radius $15^{\prime \prime}$ from a blank area of sky situated near to the source position. The count rates were obtained from the event lists using uvotevtlc and from the images using uvotsource. The used software can be found in the software release, Headas 6.3.2 and version 20071106 (UVOT) of the calibration files.

For each filter, the count rates were binned by taking the weighted average in time bins of $\Delta t / t=0.2$. They were then converted to magnitudes using the UVOT photometric zero points (Poole et al. 2008).

\subsection{Spectroscopic data}

Low-resolution integral field spectroscopy of the field was acquired starting about $7.5 \mathrm{~h}$ after the burst. Even if at that time the afterglow position was already precisely known, we decided to perform the IFS observing run, in order to learn the handling of the data.

The observations were carried out starting at UT 01:43:41 (June 6), at the 3.5-m telescope equipped with the Potsdam Multi-Aperture Spectrograph (PMAS; Roth et al. 2005) in the PPak (PMAS fiber Package) mode (Verheijen et al. 2004; Kelz et al. 2006), using $2 \times 2$ pixel binning. We used the V300 grating, which covers a wavelength range between 3698 and $7010 \AA$, resulting in a reciprocal dispersion of $3.4 \AA$ per pixel. The PPak fiber bundle consists of 382 fibers of 2 .'7 diameter each (see Fig. 5 in Kelz et al. 2006). Of them, 331 fibers (the science fibers) are concentrated in a single hexagonal bundle covering a field-of-view of $74^{\prime \prime} \times 64^{\prime \prime}$ with a filling factor of $\sim 65 \%$.

The sky is sampled by 36 additional fibers, distributed in 6 bundles of 6 fibers each, located following a circular distribution at $\sim 90^{\prime \prime}$ from the center and at the edges of the central hexagon. The sky-fibers are distributed among the science ones in the pseudo-slit, in order to have a good sampling of the sky. The remaining 15 fibers are used for calibration purposes.

Given that PMAS/PPak has a filling factor less than 1, during the observations a dithering scheme was applied. The observations consisted of 9 single exposures of $15 \mathrm{~min}$ each, i.e. 3 images for every dither pointing. As 3 of the 9 exposures had a low $\mathrm{S} / \mathrm{N}$ due to the presence of clouds, only six of them were considered. Figure 2 shows the average of the second dither pointing images, when the magnitude of the afterglow was about $R_{\mathrm{C}}=19.5$. The field of view is sampled into discrete spatial elements named SPAXELs.

The data reduction was performed twice using two different pieces of software: PPAK_online, which is part of the P3D package of IDL routines developed for the reduction of PMAS data (Becker 2002) and R3D ${ }^{3}$, a package coded in Perl by Sánchez (Sánchez \& Cardiel 2005; Sánchez 2006). In combination with the previous ones, IRAF ${ }^{4}, \mathrm{MIDAS}^{5}$ and the $\mathrm{E}^{6} \mathrm{D}^{6}$ visualization

\footnotetext{
${ }^{3}$ http://www. caha.es/sanchez/r3d/index.html

4 http://iraf.noao.edu

5 http://www. eso.org/projects/esomidas

${ }^{6}$ http://www.aip.de/Euro3D/E3D/\#Docu
}

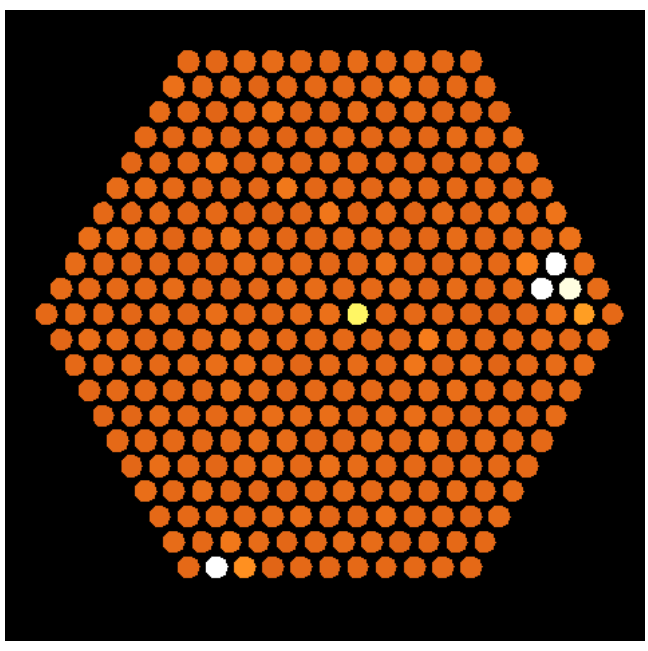

Fig. 2. The field of GRB 060605 seen by the PPak spaxels. The image is the average of three exposures when the afterglow (the yellow spot in the middle) had a magnitude of about $R_{\mathrm{C}}=19.5$. The other bright spaxels are stars (see Fig. 1).

tool (Sánchez 2004) were used. The results obtained using the two packages were consistent.

The reduction of spectroscopic data obtained with fiberbased integral-field units consists of the following standard steps: bias subtraction, flat field correction, location of the spectra on the CCD (the so-called tracing), spectra extraction, wavelength calibration, fiber flat correction, sky subtraction, cosmic ray rejection and flux calibration.

The bias frame, obtained immediately after the target frame, was cleaned and smoothed using boxsizes of 5 pixels in $x$ and $y$ to create the final bias frame. Domeflat exposures of $5 \mathrm{~s}$ were taken before and after the object observations to produce a trace mask, i.e. to locate the spectra along the cross-dispersion direction on the CCD (for a detailed description of tracing, see Becker 2002). Once this mask is defined one can easily extract the spectra from the $\mathrm{CCD}$, producing a so-called row-stackedspectra image, where one row represents one spectrum.

For wavelength calibration a combined $\mathrm{He} / \mathrm{Rb}$-emission lamp exposure of $15 \mathrm{~s}$ was obtained at the beginning of the night with the additional illumination of 15 separate calibration fibers with ThAr. Simultaneous ThAr-exposures of these calibration fibers during lamp flat and object observations as well were used to correct for flexure effects of the instrument (Kelz et al. 2006). We defined some (at least two) of the ThAr spots in the lamp flat image as reference and calculated their shifts in $x$ and $y$ versus the same ThAr spots in the object images. These shift values were taken into account during tracing, spectra extraction, and wavelength calibration as well.

For the sky subtraction the spaxels not contaminated by sources were selected and the average extracted spectrum was then subtracted from the science spectrum. For this purpose we used the E3D package (Sánchez 2004).

After cosmic ray rejection, the final spectrum was flux calibrated using the spectrophotometric standard star $\mathrm{Hz} 44$ (Oke 1990). As the spectra of the optical afterglow were extracted on one spaxel, the spectra of the standard star were extracted in the same way. A cross check on the flux calibration was performed using the observed $R_{\mathrm{C}}$-band photometric magnitude. 


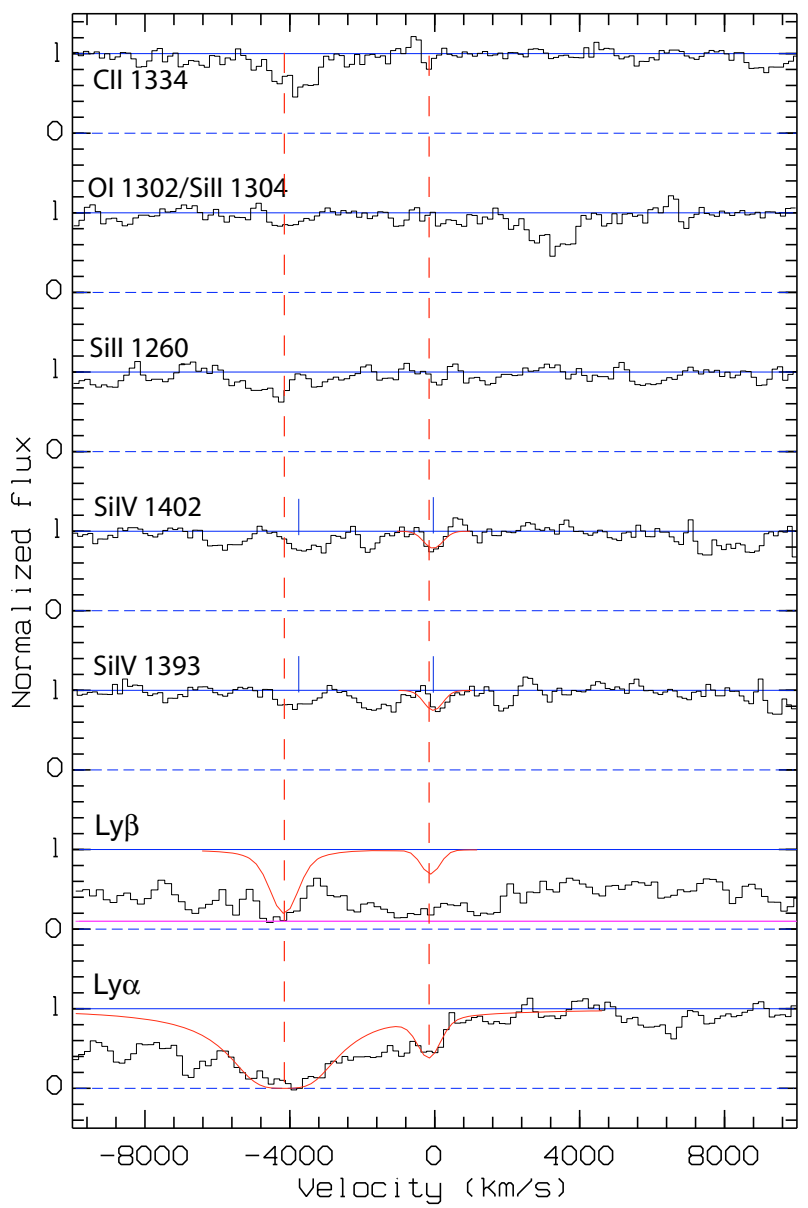

Fig. 3. The spectrum of the afterglow of GRB 060605 in velocity space, centered at a redshift of $z=3.773\left(v=0 \mathrm{~km} \mathrm{~s}^{-1}\right)$. This is likely the redshift of the GRB, for which we detect Ly $\alpha$ and Si IV doublet absorptions. Offset by about $-4000 \mathrm{~km} \mathrm{~s}^{-1}$ we also mark the strong absorption system (damped Lyman $\alpha$ ) at $z=3.709$ with Ly $\alpha$, Ly $\beta$, O I and the Si IV doublet, likely associated with an intervening galaxy. For the method see Savaglio \& Fall (2004).

\section{Results}

We first present here the results of our spectroscopy since this provided the accurate redshift information.

\subsection{The optical spectrum of the afterglow}

In Fig. 3 we show absorption lines identified in the PPak spectrum of the afterglow (spectral resolution $\lambda / \Delta \lambda=500$ at a wavelength of $5300 \AA$ ). The highest redshifted Ly $\alpha$ is at $z=$ $3.773 \pm 0.001$, which we interpret as the redshift of the GRB (look-back time $11.98 \mathrm{Gyr}$ ). The H I column density is very uncertain, in the range $N_{\mathrm{HI}}=10^{18.5}-10^{19.3} \mathrm{~cm}^{-2}$, but certainly one of the lowest ever measured in a GRB afterglow at the redshift of the GRB (Savaglio 2006; Jakobsson et al. 2006; Chen et al. 2007b). We notice that the H I column densities measured for GRB 021004 and GRB 060607A are also low, $N_{\mathrm{HI}}=10^{19.5} \mathrm{~cm}^{-2}$ and $10^{16.8} \mathrm{~cm}^{-2}$, in the former and latter, respectively. For GRB 030226, Shin et al. (2006) report $N_{\mathrm{HI}}=10^{20.5 \pm 0.3} \mathrm{~cm}^{-2}$. Possible explanations for such low H I column densities might be either the ionization of the GRB environment by the intense fireball light, the localisation of the burst source in a star-forming region including many UV-bright massive stars, or the location of the GRB progenitor in the outer part of its host galaxy.
Blueward of the $z=3.773$ we identify a strong Ly $\alpha$ absorber at $z=3.709 \pm 0.003\left(\Delta v=4000 \mathrm{~km} \mathrm{~s}^{-1}\right.$ from the GRB redshift $)$ likely associated with a Damped Ly $\alpha$ system (DLA), with an estimated H I column density of $N_{\mathrm{HI}}=10^{20.9} \mathrm{~cm}^{-2}$. Redward of the Ly $\alpha$, we detect the Si IV doublet at $z=3.717 \pm 0.001(\Delta v=$ $500 \mathrm{~km} \mathrm{~s}^{-1}$ from the DLA). At approximately the redshift of the DLA, we identify absorption lines associated with C II 1334, Si II 1260, and O I 1302/ Si II 1304 (Fig. 3). Unfortunately, the low $\mathrm{S} / \mathrm{N}$ of the spectrum does not allow us to measure column densities for metals. We also identify a strong Si IV absorption doublet at $z=3.774 \pm 0.001, \Delta v=120 \mathrm{~km} \mathrm{~s}^{-1}$ redward of the Ly $\alpha$, likely associated with the GRB-host system.

The separation between the two strong $z=3.717$ and $z=$ $3.774 \mathrm{Si}$ IV absorbers $\Delta v=3600 \mathrm{~km} \mathrm{~s}^{-1}$ is comparable to that between the double C IV absorbers detected in the afterglows of GRB $021004\left(\Delta v=2400 \mathrm{~km} \mathrm{~s}^{-1}\right.$; Savaglio et al. 2002; Fiore et al. 2005), GRB $030226\left(\Delta v=2400 \mathrm{~km} \mathrm{~s}^{-1}\right.$; Klose et al. 2004), and GRB 060607A $\left(\Delta v=1800 \mathrm{~km} \mathrm{~s}^{-1}\right.$; Smette et al., in preparation). The possibility that this is the signature of the stellar wind from the GRB progenitor (a Wolf-Rayet star) has been discussed in the literature in detail (Mirabal et al. 2003; Schaefer et al. 2003) but recently disfavoured for most cases by Chen et al. (2007a). The latter authors suggested as a reason for this effect the presence of foreground galaxies along the sight line of the GRBs. Our deep VLT imaging of the field (Sect. 4.5) does unfortunately not identify these potential absorbers with certainty. So, which of these two possibilities explains our observations of GRB 060605 remains open.

In Fig. 4, the spectrum of a Lyman break galaxy (LBG) at a $z \sim 3$ (from Shapley et al. 2001) is overplotted on the spectrum of the GRB to show the typical absorption lines observed in these galaxies at high redshifts and to help the comparison with our spectrum. The spectrum of the galaxy has been shifted in wavelength considering the redshift of the damped Lyman $\alpha$ absorption system at $z=3.709$ and rescaled in flux for comparison. While the data seem to indicate an underabundance of oxygen in the GRB host galaxy, the low $\mathrm{S} / \mathrm{N}$ ratio of our data does not allow us to draw quantitative conclusions.

\subsection{The $X$-ray afterglow}

The X-ray afterglow of GRB 060605 was detected by Swift for more than 1 day after the trigger. When analyzing the data we rebinned them by taking 30 counts/bin in order to obtain a good $\mathrm{S} / \mathrm{N}$ ratio. As already noted by Godet et al. (2006), and as it is shown in (Fig. 5), as a first guess the X-ray light curve consists only of three power-law segments. The numerical ansatz to describe the X-ray light curve is then a smoothly broken double power-law (see Liang et al. 2008). In doing so, we fixed the smoothness parameters $n_{1}$ and $n_{2}$ (in their paper $\omega_{1}$ and $\left.\omega_{2}\right)$ to -10 and 10 , in the case of transition I to II and transition II to III, respectively. This describes a sharp break. For the steep-to-shallow transition (I to II), we find a break time of $210 \pm$ $30 \mathrm{~s}(0.0024 \pm 0.0003$ days $)$, while the shallow-to-steep transition (II to III) took place at $7510 \pm 410 \mathrm{~s}(0.0869 \pm 0.0047$ days $)$ after the trigger. At the beginning, the afterglow decays with a slope of $\alpha_{\mathrm{I}}=2.19 \pm 0.42$, followed by $\alpha_{\mathrm{II}}=0.34 \pm 0.03$ during the shallow decay phase, and it continues to decay with $\alpha_{\mathrm{III}}=1.89 \pm 0.07$ $\left(\chi^{2} /\right.$ d.o.f $\left.=55.5 / 42=1.32\right)$. Within errors, these values are in agreement with the results reported by Godet et al. (2006). In Sect. 4.1 we will test if this ansatz of a three-segment X-ray light curve is compatible with the basic theoretical concepts describing afterglows. 


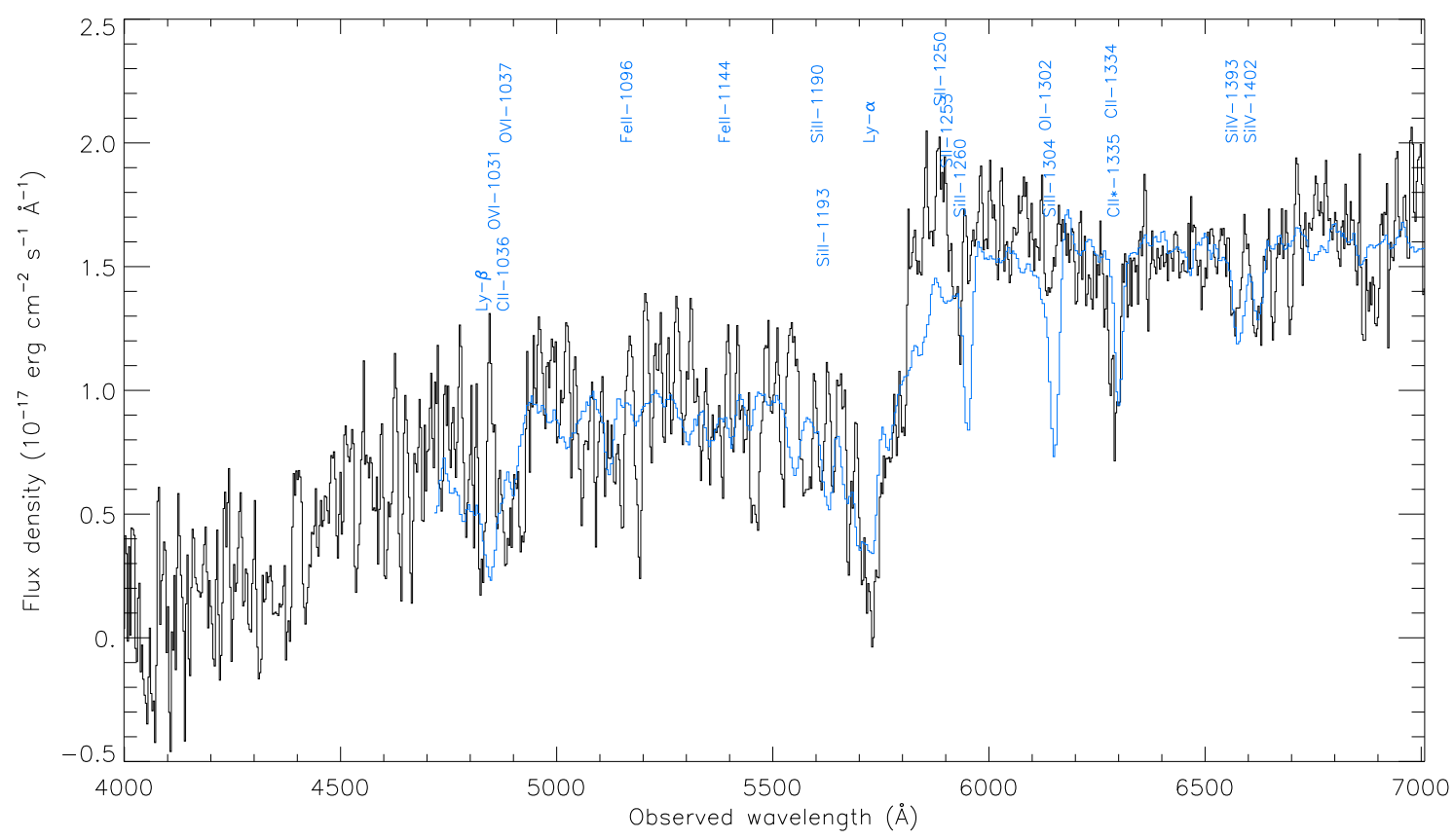

Fig. 4. Spectrum of the afterglow (black) of GRB 060605 obtained between 7.5 and $9.1 \mathrm{~h}$ after the burst with PPak mounted at the Calar Alto 3.5-m telescope, overplotted with the spectrum of a Lyman break galaxy (LBG) at the same redshift (from Shapley et al. 2001). The typical LBG lines are indicated in blue; only the strongest of them can also be found in the afterglow spectrum. The afterglow spectrum, calibrated in flux and wavelength, is a composition of six individual spectra of 15 min exposure time each. The spectral resolution is $\lambda / \Delta \lambda=500$.

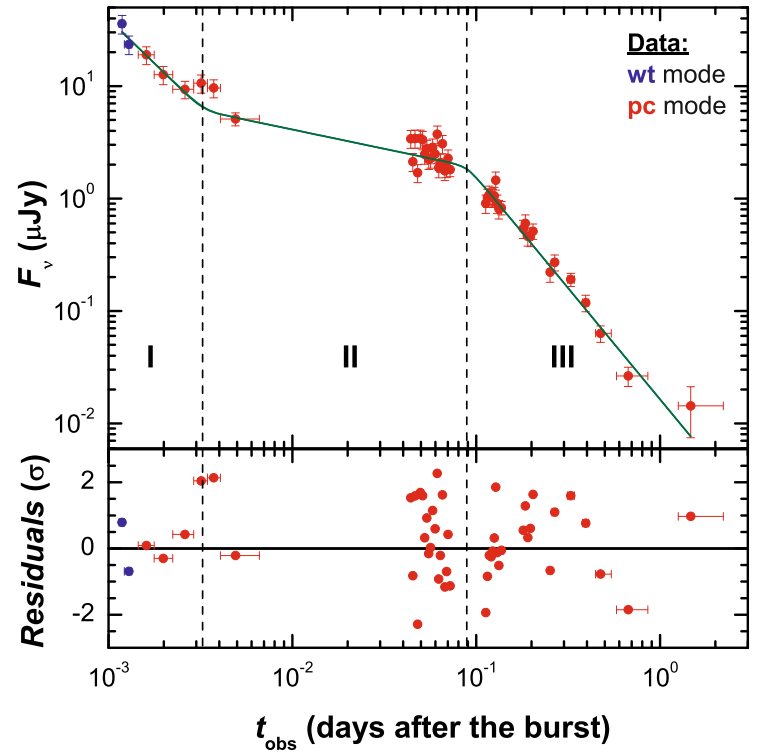

Fig. 5. The X-ray light curve of the afterglow of GRB 060605 observed with Swift XRT (Evans et al. 2007). Formally, three powerlaw segments can be distinguished (Godet et al. 2006). A small flare is seen at the beginning of the second decay phase (II). The lower panel shows the residuals of the best fit. Fluctuations in the light curve $(>2 \sigma)$ are also seen at later times. The energy conversion factor is $4.6 \times 10^{-11} \mathrm{erg} \mathrm{cm}^{-2}$ counts $^{-1}$.

After dividing the $0.3-6 \mathrm{keV}^{7}$ XRT spectrum, derived from $\mathrm{PC}$ data, in several spectra over small time intervals, as no spectral evolution was found, we took the overall spectrum between $t=126 \mathrm{~s}$ (0.0015 days) and $t=7.4 \times 10^{4} \mathrm{~s}$ (0.86 days) (Fig. 6). It is well fitted by an absorbed power-law with a spectral

7 The $0.3-10 \mathrm{keV}$ XRT spectrum had no signal in the range $6-10 \mathrm{keV}$ and for this reason only the first part was considered.

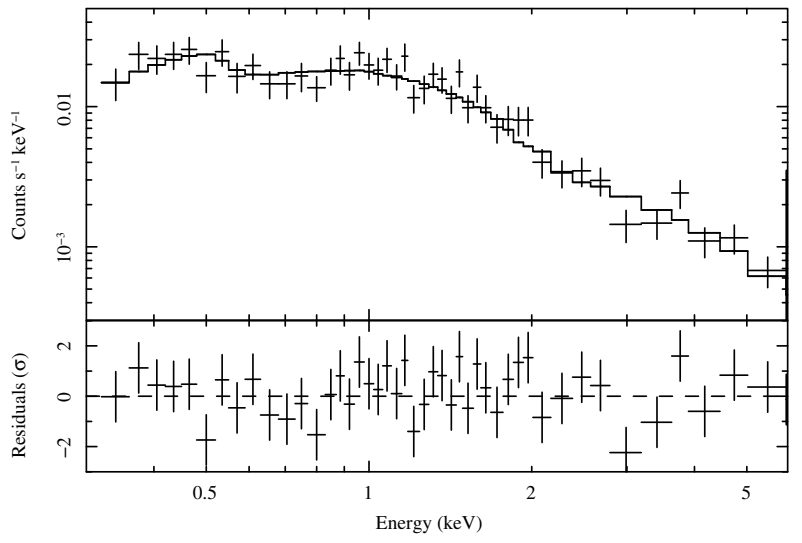

Fig. 6. X-ray spectrum of the afterglow of GRB 060605 obtained in photon counting mode between 0.0015 and 0.8565 days after the trigger. The lower panel shows the residuals of the best fit (for more details see Sect. 3.2).

index $\beta_{\mathrm{X}}=1.06 \pm 0.16\left(\chi^{2} /\right.$ d.o.f. $\left.=39.6 / 44=0.93\right)$ and $N_{\mathrm{H}}=5.5_{-2.9}^{+3.3} \times 10^{20} \mathrm{~cm}^{-2}$. Due to the big uncertainty this last value is consistent with both the Galactic hydrogen column density of $N_{\mathrm{H}}^{\mathrm{Gal}}=5.1 \times 10^{20} \mathrm{~cm}^{-2}$ by Dickey \& Lockman (1990) and the lower value of $N_{\mathrm{H}}^{\mathrm{Gal}}=4.1 \times 10^{20} \mathrm{~cm}^{-2}$ given by the recent release of the Leiden/Argentine/Bonn (LAB) Survey of Galactic H I (Kalberla et al. 2005). No additional rest frame hydrogen column density can be found in the X-ray spectrum. Adding such a component by hand did not improve the fit. The lack of evidence of additional hydrogen in the host galaxy is in agreement with the finding of Grupe et al. (2007a) that high-redshift events usually do not show such a feature.

However, due to the large uncertainty on $N_{\mathrm{H}}$ (Ly $\alpha$ ) and no strong constraints on $N_{\mathrm{H}}$ (X-ray), we cannot exclude that for GRB 060605 the optical and the X-ray data trace a different 
population of hydrogen at the redshift of the burst, as it has been found in many other cases (Watson et al. 2007).

\subsection{The UV/optical light curve and its SED}

We combined our UVOT data (Table 1) with further data reported in the GRB Coordinates Network Circulars (Rykoff \& Schaefer 2006; Schaefer et al. 2006; Khamitov et al. 2006a,b; Malesani et al. 2006; Zhai et al. 2006; Karska \& Garnavich 2006; Sharapov et al. 2006), all taken in the $R_{\mathrm{C}}$ filter, or unfiltered calibrated to the $R_{\mathrm{C}}$ band. Karska \& Garnavich (2006) point out zero-point discrepancies between different USNO $R_{\mathrm{C}}$ magnitudes, and we can confirm that the magnitudes reported by Khamitov et al. (2006a) are about one magnitude fainter than what would be expected from the joint light curve (see below), whereas the late detection by Khamitov et al. (2006b) agrees well with the steep decay slope found by Karska \& Garnavich (2006) and one additional point from Pozanenko et al., in preparation. We added an error of $0.1 \mathrm{mag}$ in quadrature to all GCN data points to account for the different filters and reference stars.

Using the $R_{\mathrm{C}}$-band light curve as the most reliable template, and correcting all data for the foreground extinction of $E_{B-V}=0.049$ (Schlegel et al. 1998), we derive colours for the UVOT detections. We find $v-R_{\mathrm{C}}=1.2 \pm 0.2$, white $-R_{\mathrm{C}}=$ $2.3 \pm 0.2, b-R_{\mathrm{C}}=2.45 \pm 0.3, u-R_{\mathrm{C}}>3.3$, uvw $1-R_{\mathrm{C}}>2.5$, uvm $2-R_{\mathrm{C}}>4.5$, and $u v w 2-R_{\mathrm{C}}>2.5$. We note that, usually, white magnitudes are close in value to UVOT $v$-band values. Given the high redshift of the source, however, the large white $-v$ colour is due to the unfiltered UVOT bandpass being strongly affected by Lyman damping, making the afterglow much redder than usual (see below). We used the derived colour indices to shift the UVOT detections ( $v$, white and $b$ ) to the $R_{\mathrm{C}}$-band and to construct a composite light curve (Fig. 7).

In the UV/optical bands the data are broadly consistent with an achromatic evolution, but we caution that the data are sparse. We find an early rise, as reported by others (Schaefer et al. 2006; Zhai et al. 2006), which is followed by a "classical" broken power-law decay. Denoting the three slopes $\alpha_{R}$ (where the index $R$ stands for rise), $\alpha_{1}$ and $\alpha_{2}$, we find $\alpha_{R}=-0.70 \pm 0.15$, $\alpha_{1}=0.89 \pm 0.04$ and $\alpha_{2}=2.58 \pm 0.15$. The break times are $0.0044 \pm 0.0007$ days for the break from rise to decay, and $0.27 \pm 0.02$ days for the second break. In both cases, we assumed that the host galaxy underlying the afterglow has an extinctioncorrected magnitude of $R_{\mathrm{C}}=26.3$ (see Sect. 4.5). We fixed the break smoothness parameter $n_{2}$ according to Liang et al. (2008) to 10 for the second break. For the first break, while we were not able to leave $n_{1}$ as a free parameter of the fit, we find a minimum $\chi^{2}$ and a very good fit $\left(\chi^{2} /\right.$ d.o.f. $\left.=21.00 / 32=0.66\right)$ for a rather smooth break $n_{1}=2.5$. A summary of the fit parameters is given in Table 3.

The peak time of $358 \pm 61 \mathrm{~s}(0.0041 \pm 0.0007$ days $)$ can be found from the light curve fit by setting $\mathrm{d} F_{v}(t) / \mathrm{d} t=0$ and has a value that is comparable to what has been found for, e.g., the early phase of the optical afterglow of GRB 060418 and 060607A (Molinari et al. 2007). Our result for $\alpha_{1}$ is in agreement with the value reported by Schaefer et al. (2006) and the peak time we derive is in agreement with Zhai et al. (2006).

The afterglow of GRB 060605 belongs to the growing ensemble of optical afterglows for which thanks to a rapid response in the follow-up observations the data show the early rise of the afterglow, as predicted by theoretical models (Panaitescu \& Kumar 2000; Sari 1997). With a peak magnitude of $R_{\mathrm{C}}=15.2$ at $t \approx 360 \mathrm{~s}$ (0.0042 days) (Fig. 7) it is among the brightest optical afterglows ever detected (Nardini et al. 2008; Kann et al. 2007).
Table 1. Log of the Swift UVOT observations.

\begin{tabular}{|c|c|c|c|c|}
\hline$t$ & $\Delta t-$ & $\Delta t+$ & Magnitude & Filter \\
\hline 106.6 & 4.9 & 5.1 & $18.23_{-0.16}^{+0.18}$ & white \\
\hline 126.6 & 4.9 & 5.1 & $17.97_{-0.13}^{-0.110}$ & white \\
\hline 151.6 & 7.3 & 7.7 & $18.06_{-012}^{+0.13}$ & white \\
\hline 181.5 & 7.3 & 7.6 & $17.83_{-0.10}^{+0.11}$ & white \\
\hline 4690 & 49 & 49 & $19.81_{-0.14}^{+0.10}$ & white \\
\hline 6122 & 49 & 49 & $20.14_{-0.24}^{-0.034}$ & white \\
\hline 17560 & 193 & 195 & $20.90_{-0.23}^{+0.34}$ & white \\
\hline 85.7 & 7.6 & 8.4 & $17.72 \pm 0.66$ & $v$ \\
\hline 220 & 9 & 9 & $16.66_{-0.14}^{+0.16}$ & $v$ \\
\hline 263 & 12 & 13 & $16.69_{-012}^{+0.14}$ & $v$ \\
\hline 318 & 15 & 15 & $16.37_{-0.09}^{-0.10}$ & $v$ \\
\hline 383 & 17 & 18 & $16.67_{-0.11}^{-0.09}$ & $v$ \\
\hline 463 & 22 & 23 & $16.45_{-0.09}^{+0.10}$ & $v$ \\
\hline 551 & 21 & 22 & $16.60_{-0.10}^{+0.12}$ & $v$ \\
\hline 5100 & 49 & 49 & $18.45_{-0.18}^{+0.22}$ & $v$ \\
\hline 10013 & 223 & 228 & $19.33_{-0.13}^{+0.14}$ & $v$ \\
\hline 34052 & 225 & 226 & $>20.57$ & $v$ \\
\hline 51412 & 225 & 226 & $>20.58$ & $v$ \\
\hline 68768 & 225 & 226 & $>20.53$ & $v$ \\
\hline 116954 & 8680 & 8845 & $>21.40$ & $v$ \\
\hline 184147 & 3318 & 3379 & $>21.32$ & $v$ \\
\hline 4486 & 49 & 49 & $20.10_{-0.22}^{+0.27}$ & $b$ \\
\hline 5918 & 49 & 49 & $20.20_{-0.30}^{+0.41}$ & $b$ \\
\hline 16711 & 224 & 227 & $21.06_{-0.24}^{+0.30}$ & $b$ \\
\hline 23377 & 177 & 179 & $21.27_{-0.40}^{+0.65}$ & $b$ \\
\hline 40696 & 197 & 198 & $>21.02$ & $b$ \\
\hline 58053 & 395 & 396 & $>20.98$ & $b$ \\
\hline 4281 & 49 & 49 & $>20.39$ & $u$ \\
\hline 5713 & 49 & 49 & $>20.22$ & $u$ \\
\hline 15799 & 224 & 227 & $>21.89$ & $u$ \\
\hline 22560 & 224 & 227 & $>21.57$ & $u$ \\
\hline 29221 & 148 & 149 & $>20.19$ & $u$ \\
\hline 43093 & 1831 & 1913 & $>22.68$ & $u$ \\
\hline 60462 & 1843 & 1901 & $>21.79$ & $u$ \\
\hline 4077 & 49 & 49 & $>19.87$ & $u v w 1$ \\
\hline 5509 & 49 & 49 & $>19.27$ & $u v w 1$ \\
\hline 11768 & 191 & 194 & $>19.92$ & $u v w 1$ \\
\hline 21645 & 220 & 222 & $>21.54$ & $u v w 1$ \\
\hline 28460 & 220 & 222 & $>20.90$ & $u v w 1$ \\
\hline 42229 & 1852 & 1937 & $>21.28$ & $u v w 1$ \\
\hline 59599 & 1864 & 1925 & $>22.37$ & $u v w 1$ \\
\hline 73712 & 221 & 221 & $>21.22$ & $u v w 1$ \\
\hline 3872 & 49 & 49 & $>21.95$ & uvm 2 \\
\hline 5304 & 49 & 49 & $>21.85$ & uvm 2 \\
\hline 10916 & 219 & 223 & $>21.58$ & uvm 2 \\
\hline 27553 & 220 & 222 & $>20.93$ & uvm 2 \\
\hline 34905 & 195 & 196 & $>21.36$ & uvm 2 \\
\hline 48416 & 2051 & 2142 & $>22.44$ & uvm 2 \\
\hline 65785 & 2063 & 2130 & $>21.52$ & uvm 2 \\
\hline 4895 & 48 & 49 & $>20.19$ & uvw2 \\
\hline 6304 & 37 & 37 & $>20.18$ & $u v w 2$ \\
\hline 33138 & 221 & 222 & $>21.59$ & uvw2 \\
\hline 50495 & 220 & 221 & $>21.24$ & $u v w 2$ \\
\hline 67853 & 221 & 222 & $>22.16$ & $u v w 2$ \\
\hline
\end{tabular}

The first column gives the logarithmic mid-time in seconds after the onset of the GRB. The second and the third columns give the start/end of the observations. The data are not corrected for Galactic extinction. 


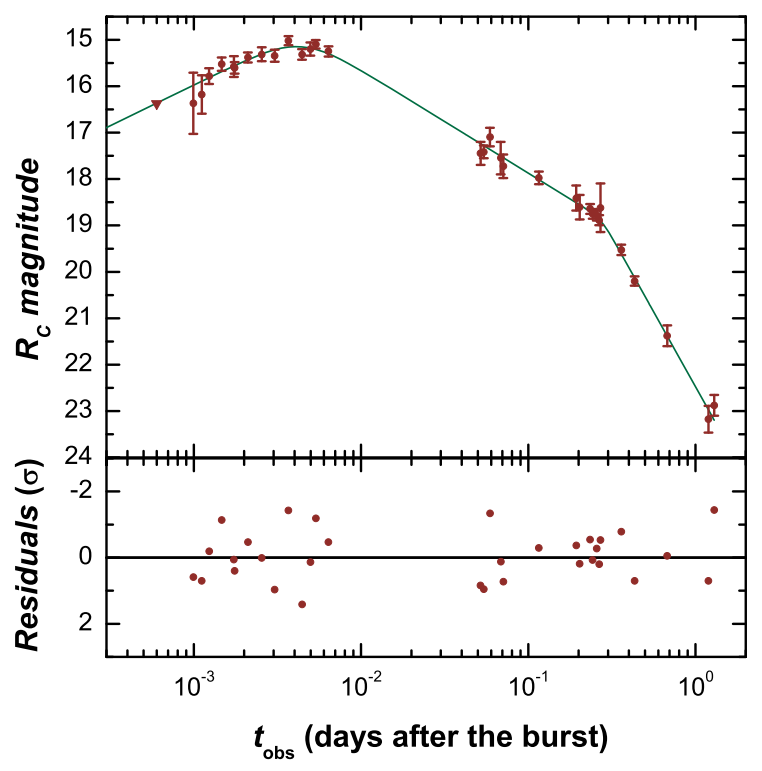

Fig. 7. The composite $R_{\mathrm{C}}$-band light curve of the afterglow of GRB 060605 (see text for more details), fitted with a double broken power-law. The lower panel gives the residuals of the fit. These data are corrected for Galactic extinction. See the text for the results of the fit.

Table 2. Values plotted in Fig. 8.

\begin{tabular}{lcccc}
\hline \hline Filter & $\begin{array}{c}\lambda \\
(\AA)\end{array}$ & $\begin{array}{c}v(1+z) \\
\left(10^{15} \mathrm{~Hz}\right)\end{array}$ & mag & $\begin{array}{c}F_{v} \\
(\mu \mathrm{Jy})\end{array}$ \\
\hline$R_{\mathrm{C}}$ & 6588 & 2.17 & $18.83 \pm 0.04$ & $90.40 \pm 3.33$ \\
$v$ & 5402 & 2.65 & $20.03 \pm 0.2$ & $35.38 \pm 6.52$ \\
$b$ & 4329 & 3.31 & $21.28 \pm 0.3$ & $13.09 \pm 3.62$ \\
$u$ & 3501 & 4.09 & $>22.13$ & $<2.54$ \\
$u v w 1$ & 2634 & 5.43 & $>21.33$ & $<2.56$ \\
$u v m 2$ & 2231 & 6.41 & $>23.33$ & $<0.41$ \\
uvw2 & 2030 & 7.05 & $>21.33$ & $<2.82$ \\
\hline
\end{tabular}

The $u, u v w 1, u v m 2$ and $u v w 2$ data are upper limits. For all filters except $R_{\mathrm{C}}, \lambda$ is from Poole et al. (2008). Data refer to $t=0.27$ days. A redshift of $z=3.773$ was assumed. The values have been corrected for Galactic extinction. The fluxes have been calculated assuming the conversion factors from Bessell (1979) and Poole et al. (2008).

Table 3. Parameters of the fit of the optical afterglow light curve (Fig. 7).

\begin{tabular}{ll}
\hline \hline Parameter & Value \\
\hline$\alpha_{\mathrm{R}}$ & $-0.70 \pm 0.15$ \\
$\alpha_{1}$ & $0.89 \pm 0.04$ \\
$\alpha_{2}$ & $2.58 \pm 0.15$ \\
$t_{1}$ (days) & $0.0044 \pm 0.0007$ \\
$t_{2}$ (days) & $0.27 \pm 0.02$ \\
$n_{1}$ & 2.5 \\
$n_{2}$ & 10 \\
$\chi^{2} /$ d.o.f. & $21.00 / 32=0.66$ \\
\hline
\end{tabular}

The slope $\alpha_{R}$ describes the rising part of the light curve, $\left(t_{1}, n_{1}\right)$ and $\left(t_{2}, n_{2}\right)$ describe the two breaks.

Using the colours derived above, we can construct the spectral energy distribution (SED; Fig. 8 and Table 2) at $t=$ 0.27 days. We have detections in only three filters $\left(B V R_{\mathrm{C}}\right)$. These three data points can be fit with a very steep SED: we find $\beta_{\text {opt }}=4.64 \pm 0.58$. The steep slope is further confirmed by the $u$-band and $u v w 2$-band upper limits, the other two filters are less constraining. This is much steeper than typical afterglow

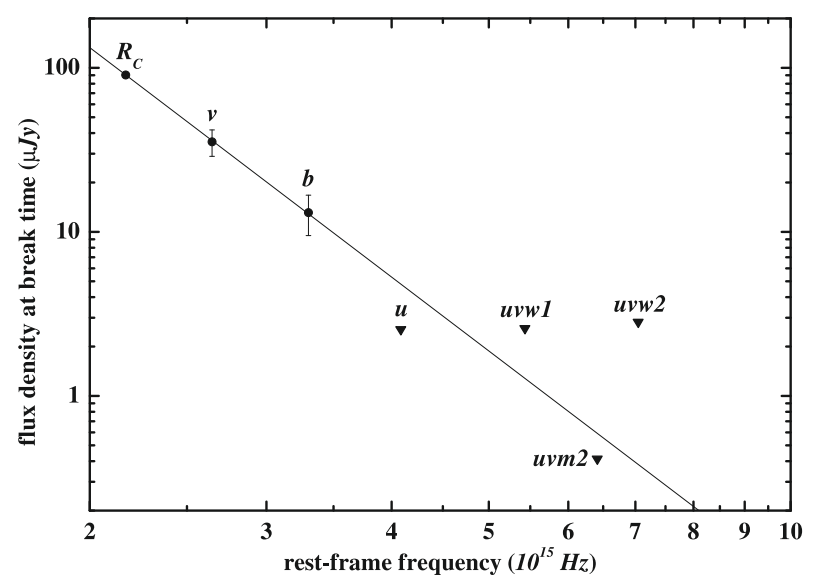

Fig. 8. Spectral energy distribution (SED) of the afterglow of GRB 060605 in the optical bands at $t=0.27$ days. The line is the bestfit power law. Detections in three filters $\left(b v R_{\mathrm{C}}\right)$ and deep upper limits in $u$ and in $u v m 2$ show a very steep SED $\left(\beta_{\text {opt }}=4.64 \pm 0.58\right)$, a result of Lyman blanketing in the optical/UV due to the high redshift.

slopes, which lie in the range from 0.5 to 1.1 (e.g., Kann et al. 2006, 2007). This is mostly due to the Lyman blanketing blueward of the rest-frame Ly $\alpha$ line, which at $z=3.773$ falls between the observed $v$ and $R_{\mathrm{C}}$ bands.

\section{Discussion}

\subsection{The $X$-ray light curve}

According to Nousek et al. (2006) and Zhang et al. (2006), the $\mathrm{X}$-ray light curve of an afterglow has a canonical shape. It consists of four well-defined evolutionary phases: a steep initial decay followed by a flat (plateau) phase, then a steeper decay (a pre-jet break phase) and finally a post-jet break phase (e.g., GRB 050315; Vaughan et al. 2006; Panaitescu 2008; Liang et al. 2008). However, as we have already emphasized, as a first guess the data seem to imply that the X-ray light curve of GRB 060605 consists of three power-law segments only (Fig. 5). Let us first discuss if this allows a satisfactory explanation of the observational data within the context of the fireball model. For this reason, let as discuss the nature of the second break seen in the fitted X-ray light curve at around 0.09 days. Figure 5 suggests to consider two cases.

Case A: If this is the jet break, segment II of our Fig. 5 is the pre-break segment, while segment III is the post-break phase. Consequently the slopes of segment II $\left(\alpha_{\mathrm{II}}\right)$ and of segment III $\left(\alpha_{\mathrm{III}}\right)$ are the pre-jet break decay and the post-break decay slopes, usually designated as $\alpha_{1}$ and $\alpha_{2}$, respectively.

Case B: The second possibility is that the jet break was in fact at much later times, i.e. at $\gtrsim 1.2$ days and $\alpha_{1}=\alpha_{\mathrm{III}}$. Indeed, most X-ray afterglows do not show jet breaks (Liang et al. 2007), with the most extreme example being GRB 060729 (Grupe et al. 2007b).

If indeed one of these two possibilities is compatible with the theoretical framework can be discussed first based on the $\alpha-\beta$ relations (cf. Zhang \& Mészáros 2004). For this reason, we considered the standard wind and ISM models for the isotropic case as well as for a jet with the cooling frequency $v_{\mathrm{c}}$ below and above the observers window. In addition, we also considered models with a power-law index of the electron distribution function of 
Table 4. Predicted spectral slope $\beta$ in the X-ray band for the two different cases A and B discussed in Sect. 4.1.

\begin{tabular}{clcc}
\hline \hline Model & $\beta(\alpha)$ & $\beta_{\mathrm{A}}$ & $\beta_{\mathrm{B}}$ \\
\hline Fast cooling $\left(v_{\mathrm{c}}<v_{\mathrm{X}}\right)$ & & & \\
ISM $_{\text {iso }}$ & $\left(2 \alpha_{1}+1\right) / 3$ & $0.56 \pm 0.02$ & $1.59 \pm 0.05$ \\
ISM $_{\text {jet }}$ & $\alpha_{2} / 2$ & $0.94 \pm 0.04$ & $1.52 \pm 0.15$ \\
wind $_{\text {iso }}$ & $\left(2 \alpha_{1}+1\right) / 3$ & $0.56 \pm 0.02$ & $1.59 \pm 0.05$ \\
wind $_{\text {jet }}$ & $\alpha_{2} / 2$ & $0.94 \pm 0.04$ & $1.52 \pm 0.15$ \\
Slow cooling $\left(v_{\mathrm{c}}>v_{\mathrm{X}}\right)$ & & & \\
ISM $_{\text {iso }}$ & $2 \alpha_{1} / 3$ & $0.23 \pm 0.02$ & $1.26 \pm 0.05$ \\
ISM $_{\text {jet }}$ & $\left(\alpha_{2}-1\right) / 2$ & $0.44 \pm 0.04$ & $1.02 \pm 0.15$ \\
wind $_{\text {iso }}$ & $\left(2 \alpha_{1}-1\right) / 3$ & $-0.11 \pm 0.02$ & $0.93 \pm 0.05$ \\
wind $_{\text {jet }}$ & $\left(\alpha_{2}-1\right) / 2$ & $0.44 \pm 0.04$ & $1.02 \pm 0.15$ \\
\hline
\end{tabular}

The calculated spectral slopes $\beta_{\mathrm{A}}$, and $\beta_{\mathrm{B}}$ have to be compared with the observed $\beta_{\mathrm{X}}=1.06 \pm 0.16$ (Sect. 3.2). For Case A it is $\alpha_{1}=0.34 \pm 0.03$ and $\alpha_{2}=1.89 \pm 0.07$, while for Case B it is $\alpha_{1}=1.89 \pm 0.07$ and $\alpha_{2}=3.03 \pm 0.30$.

less than 2, as they are listed in table 1 of Zhang \& Mészáros (2004). Based on our data the latter models turned out to be excluded with high significance, however. So, we followed Greiner et al. (2003, their Table 6) and present here the results for the eight standard cases.

Table 4 shows the predicted values for the spectral slope $\beta$ for the considered scenarios as a function of the observed light curve decay slope in the X-ray band. These results have to be compared with the observed $\beta$ in the X-ray band, $\beta_{\mathrm{X}}=1.06 \pm 0.16$ (Sect. 3.2). For Case B we calculated $\alpha_{2}$ via $\alpha_{2}=\alpha_{1}+1.14$, with the latter being the mean value for $\Delta \alpha$ in our data base of GRB afterglows with an error of 0.3 to be very conservative. Table 4 shows that Case $\mathrm{A}$ is ruled out with high significance: it predicts a spectral slope before the break time (i.e., for the isotropic case) which is in clear disagreement with the observational data. This holds for the slow as well as for the fast cooling case. Case B suggests that in the X-ray band the afterglow was in the slow-cooling regime, and the wind as well as the ISM model is in agreement with the data. However, as we will discuss in the following, even this case is disfavoured if one considers other theoretical criteria.

\subsection{The broad-band SED}

In order to better distinguish among possible scenarios for the afterglow emission of GRB 060605 in the context of the standard fireball model, we studied the broad-band spectrum from the X-rays $(0.3-6 \mathrm{keV})$ to the optical $\left(R_{\mathrm{C}}\right.$ band) at two epochs. The first epoch, at 0.07 days (Fig. 9), was chosen because it is before any suspected X-ray jet break time, while the second epoch, at 0.43 days (Fig. 10), corresponds to the time of the $R_{\mathrm{C}}$-band measurement by the Nordic Optical Telescope (NOT; Sharapov et al. 2006).

The $R_{\mathrm{C}}$-band magnitude at 0.07 days was derived from UVOT data with the procedure described in Sect. 3.3. In order to check the reliability of this method, we computed in the same way the $R_{\mathrm{C}}$ magnitude at 0.43 days, finding a value fully consistent with the NOT measurement. The $R_{\mathrm{C}}$-band fluxes were corrected for extinction in our Galaxy. The 0.3 to $6 \mathrm{keV}$ spectra were derived in the following way: we deconvolved the average XRT count spectrum by assuming the best-fit power-law model, corrected it for the measured column density (Sect. 3.2), and rescaled it to the two epochs by using the multi-broken power-law model that fitted the XRT light curve best. The fit of the X-ray spectrum alone, with $N_{\mathrm{H}}$ fixed at the Galactic value

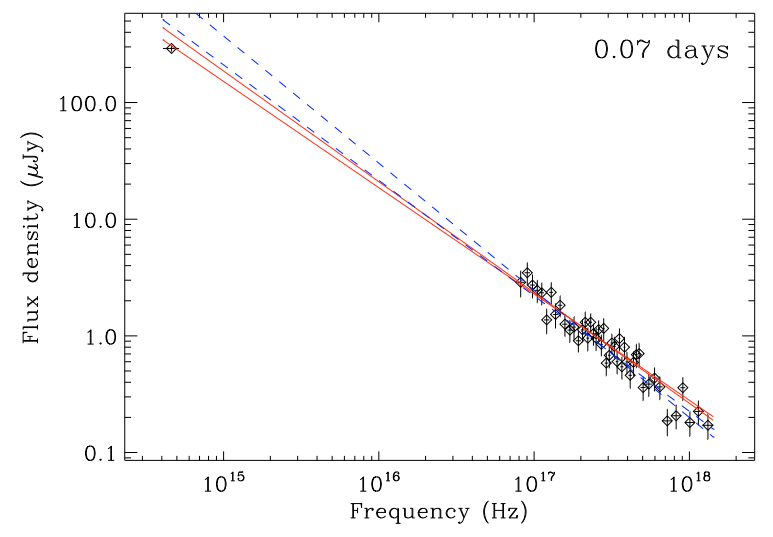

Fig.9. Broad-band spectrum of the afterglow of GRB 060605 at 0.07 days. The lines show the $1-\sigma$ confidence regions of power-law fits. Solid lines: simultaneous fit of the $\mathrm{X}$-ray and the $R_{\mathrm{C}}$-band data; dashed lines: fit of the X-ray data only. The $R_{\mathrm{C}}$-band data point was calculated from the UVOT data (Sect. 3.3). It lies beneath the spectral slope extrapolated from the X-ray band, indicating that the cooling frequency is in between the optical and the X-ray band.

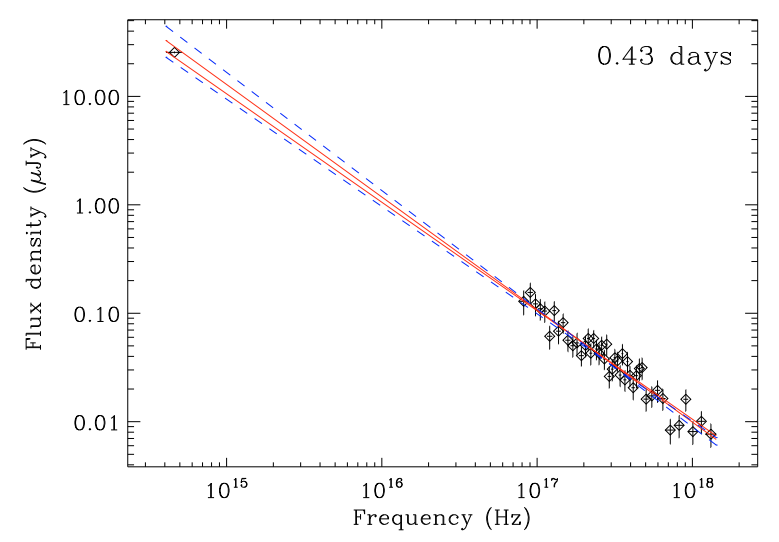

Fig. 10. The same as Fig. 9 but at 0.43 days. Here, the $R_{\mathrm{C}}$-band data point is from Sharapov et al. (2006). A simultaneous fit of the X-ray and the $R_{\mathrm{C}}$-band data gives the same result as a fit of the $\mathrm{X}$-ray data only, suggesting a change of the SED compared to 0.07 days (Fig. 9).

of $5.1 \times 10^{20} \mathrm{~cm}^{-2}$, gives a spectral index of $1.04 \pm 0.05$. Both, $\mathrm{X}$-ray and $R_{\mathrm{C}}$-band fluxes, were converted into flux densities $(\mu \mathrm{Jy})$, in order to build-up the SED and to allow for broad-band spectral fitting.

As it can be seen in Figs. 9 and 10, there is a hint of a spectral evolution between the two epochs. At 0.43 days the $R_{\mathrm{C}}$-band flux density is fully consistent with the extrapolation of the powerlaw fit of the X-ray spectrum. This is confirmed by a fit of the SED from the optical ( $R_{\mathrm{C}}$-band) to the X-ray band with a simple power-law, which provides an acceptable chi-square value $(39.5 / 44=0.90)$ and a spectral index of $\beta_{\mathrm{opt}, \mathrm{X}}=1.02 \pm 0.02$. This is evidence that at this epoch the cooling frequency, $v_{\mathrm{c}}$, was already lower than the $R_{\mathrm{C}}$-band one. On the other hand, in the spectrum at 0.07 days the $R_{\mathrm{C}}$-band data point is below the extrapolation of the power-law fit of the X-ray spectrum. Thus, at 0.07 days the cooling frequency was still at slightly higher frequencies than the $R_{\mathrm{C}}$ band, suggesting that in both spectra $v_{\mathrm{c}}$ is below the X-ray band (i.e., fast cooling regime at X-rays) and decreasing with time. The latter points to an ISM environment (Sari et al. 1998), since for a wind model $v_{\mathrm{c}}$ is increasing with time (Chevalier \& Li 2000). The former excludes even Case B (Sect. 4.1), since the $\alpha-\beta$ relations are not in agreement with the fast cooling regime in the X-ray band. Moreover, given that 


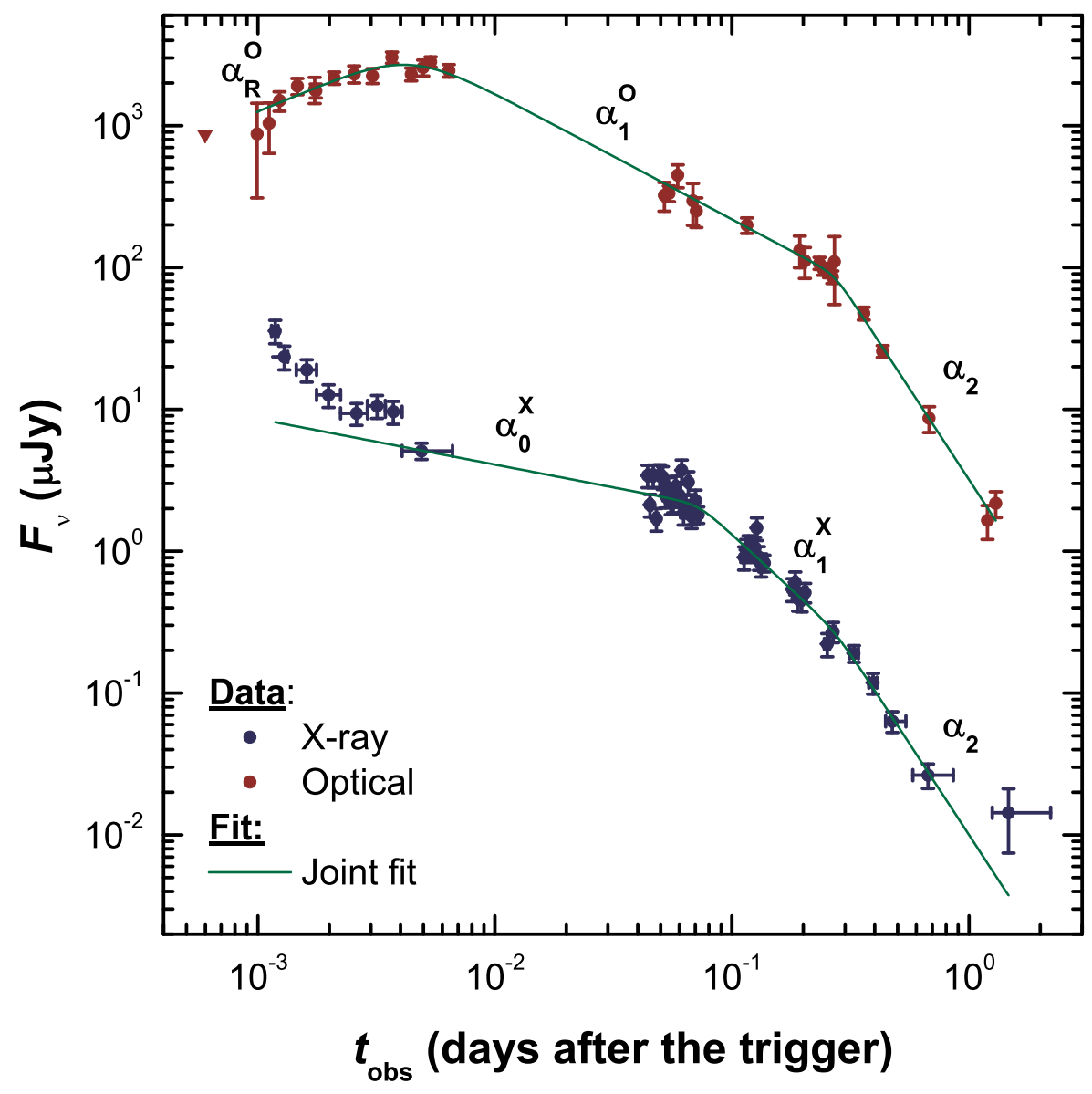

Fig. 11. The composite optical and X-ray light curve of the afterglow of GRB 060605. The final $\mathrm{X}$-ray point is an upper limit. The green line shows the results of the joint fit of the optical and the X-ray light curve (see Sect. 4.3). Note that the joint fit included only the X-ray data from $t>0.004$ days. at $t=0.43$ days the $R_{\mathrm{C}}$-band data point of the afterglow light curve lies exactly on the SED derived in the X-ray band (with a slope of $\beta_{\mathrm{X}}=1.02 \pm 0.02$; see Fig. 10), the power-law index $p$ of the electron distribution function is $p=2 \beta=2.04 \pm 0.04$, a value close to the observed mean (cf. Kann et al. 2006; see also Starling et al. 2008). If the cooling frequency were at higher values than the X-ray band ones, then $p=2 \beta+1=3.04 \pm 0.04$, an unusually large number. Consequently, the data disfavour Case $\mathrm{B}$ and thus the hypothesis of a jet break occurring after about 1.2 days.

To summarize, neither Case A or Case B lead to a reasonable agreement with the theoretical framework. While one could argue that this points to a problem with the theory, we suggest that the most reasonable hypothesis is to assume that in fact our ansatz of a three-segment X-ray light curve is incorrect and a forth power-law segment is needed. This hypothesis is motivated by the observations that many, if not most, X-ray light curves can be described by a canonical shape (Nousek et al. 2006; Zhang et al. 2006). In fact, the existence of a 4th power-law segment in the X-ray light curve is also supported by an F-test. It shows that a fit of the X-ray light curve improves if actually two breaks exist after 0.004 days instead of just one, and that the 4 th powerlaw segment is placed between about 0.07 and 0.27 days. The goodness of fit, $\chi^{2} /$ d.o.f., improves from $54.21 / 39=1.39$ to $50.74 / 37=1.18$ if an analytical ansatz is made that allows for the occurrence of two breaks after 0.004 days. This translates into a significance for an additional break of $2.3 \sigma$, or a $2 \%$ probability to find such an additional break by chance. In order to improve the fit further, we finally performed a joint fit of the optical and X-ray data.

\subsection{The X-ray vs. the optical light curve: a joint fit}

Figure 11 shows the combined optical/X-ray light curve of the afterglow of GRB 060605. At early times, from about 0.0012 to 0.0046 days, the X-ray and the optical light curves show a completely different behaviour. The X-ray light curve is falling while the optical light curve is rising, similar to what was observed for e.g. GRB 060418 (Jin \& Fan 2007). This rising optical component ends approximately at the same time as the plateau phase commences in X-ray band. The optical light curve might also include a plateau phase lasting for at least $100 \mathrm{~s}$ (0.0012 days) around the peak time.

The later behaviour of the light curve is difficult to interpret with certainty due to the lack of X-ray as well as optical data between about 0.006 and 0.041 days. Potentially, also the optical light curves could show a long-lasting plateau phase in this period if its peak was followed by a faster decay. But in this case there are no data published to check this hypothesis.

In doing the joint fit, we set the following boundary conditions: (a) an identical decay slope of the optical and the X-ray light curve and (b) an identical jet break time. In addition, as discussed before, the numerical ansatz included two breaks in the X-ray light curve after 0.004 days. For the fit the optical data of Khamitov et al. (2006a) have been excluded since they are roughly one magnitude too faint (Karska \& Garnavich 2006). The data from 0.05 to 0.1 days are the UVOT white, $b$ and $v$ measures shifted to the $R_{\mathrm{C}}$-band zero point using the early $R_{\mathrm{C}}$ and UVOT observations, and thus may be incorrect if a strong colour change occurred in between. However, no sign of a strong chromatic evolution is detected. 
Table 5. Fit parameters of the joint optical and X-ray fit $\left(\chi^{2} /\right.$ d.o.f. $=$ $62.11 / 65=0.96)$.

\begin{tabular}{ll}
\hline \hline \multicolumn{2}{c}{ Before the jet break } \\
Parameter & Value \\
\hline$\alpha_{R}^{\text {opt }}$ & $-0.70 \pm 0.15$ \\
$\alpha_{1}^{\mathrm{opt}}$ & $0.89 \pm 0.04$ \\
$t_{\mathrm{b}, 1}^{\text {opt }}($ days $)$ & $0.004 \pm 0.0007$ \\
$n_{1}^{\mathrm{opt}}$ & 2.5 \\
$\alpha_{0}^{\mathrm{X}}$ & $0.32 \pm 0.06$ \\
$\alpha_{1}^{\mathrm{X}}$ & $1.54 \pm 0.11$ \\
$t_{\mathrm{b}, 1}^{X}($ days $)$ & $0.072 \pm 0.008$ \\
$n_{1}^{\mathrm{X}}$ & 10 \\
\multicolumn{2}{c}{ After the jet break } \\
Parameter & Value \\
$\alpha_{2}$ & $2.56 \pm 0.13$ \\
$t_{\mathrm{b}, 2}($ days $)$ & $0.27 \pm 0.02$ \\
$n_{2}$ & 10 \\
\hline
\end{tabular}

Table 6. Predicted spectral slope $\beta$ considering the parameters listed in Table 5 for different applied models. The spectral slopes have to be compared with the observed $\beta_{\mathrm{X}}=1.06 \pm 0.16$ (Sect. 3.2).

\begin{tabular}{lll}
\hline \hline Model & $\beta(\alpha)$ & value \\
\hline \multicolumn{2}{l}{ Fast cooling } & \\
ISM $_{\text {iso }}$ & $\left(2 \alpha_{1}^{\mathrm{X}}+1\right) / 3$ & $1.36 \pm 0.07$ \\
ISM $_{\text {jet }}$ & $\alpha_{2} / 2$ & $1.28 \pm 0.07$ \\
wind $_{\text {iso }}$ & $\left(2 \alpha_{1}^{\mathrm{X}}+1\right) / 3$ & $1.36 \pm 0.07$ \\
wind $_{\text {jet }}$ & $\alpha_{2} / 2$ & $1.28 \pm 0.07$ \\
Slow cooling $^{\mathrm{I}}$ & \\
ISM $_{\text {iso }}$ & $2 \alpha_{1}^{\mathrm{X}} / 3$ & $1.03 \pm 0.07$ \\
ISM $_{\text {jet }}$ & $\left(\alpha_{2}-1\right) / 2$ & $0.78 \pm 0.07$ \\
wind $_{\text {iso }}$ & $\left(2 \alpha_{1}^{\mathrm{X}}-1\right) / 3$ & $0.69 \pm 0.07$ \\
wind $_{\text {jet }}$ & $\left(\alpha_{2}-1\right) / 3$ & $0.78 \pm 0.07$ \\
\hline
\end{tabular}

Allowing for a different pre-break decay slope in the optical and in the X-ray band, the joint fit finds a break time of $0.27 \pm 0.02$ days, a pre-break decay slope in the optical of $0.89 \pm 0.04$, a pre-break decay slope in the X-ray band of $1.54 \pm 0.11$, and a post-break decay slope of $2.56 \pm 0.13$ (Fig. 11 and Table 5). During the fit, the smoothness parameters were always fixed. Applying the $\alpha-\beta$ relations now shows that a wind model is disfavoured. This is in agreement with our results in Sect. 4.2, where we found that the cooling frequency was decreasing with time, indicating in this way an expansion of the fireball into a circumburst medium with an ISM profile. A decision between fast and slow cooling cannot be made, however. Both are acceptable within $2 \sigma$. Given that we know already from our analysis of the SED that the X-ray emitting electrons were in the fast cooling region at 0.07 days after the burst (Sect. 4.2), one might wonder if an agreement between theory and observations at just the $2 \sigma$ level is acceptable at all. We believe that the data quality is simply not good enough in order to obtain more robust results. At least it is obvious that the joint fit in combination with a 4-segment X-ray light curve provides a much better explanation of the observational data than a simple 3-segment ansatz (Sect. 4.1).

We can now use the observational data to constrain the density $n$ in the circumburst medium and the parameter $\varepsilon_{B}$ that measures the fraction of energy carried by the magnetic field. For an ISM medium the cooling frequency is given by (cf. Granot et al. 2000)

$v_{\mathrm{c}}=2.9 \times 10^{15} \varepsilon_{B,-1}^{-3 / 2} E_{52}^{-1 / 2} n_{0}^{-1} t_{2}^{-1 / 2}(1+z)^{-1 / 2} \mathrm{~Hz}$,

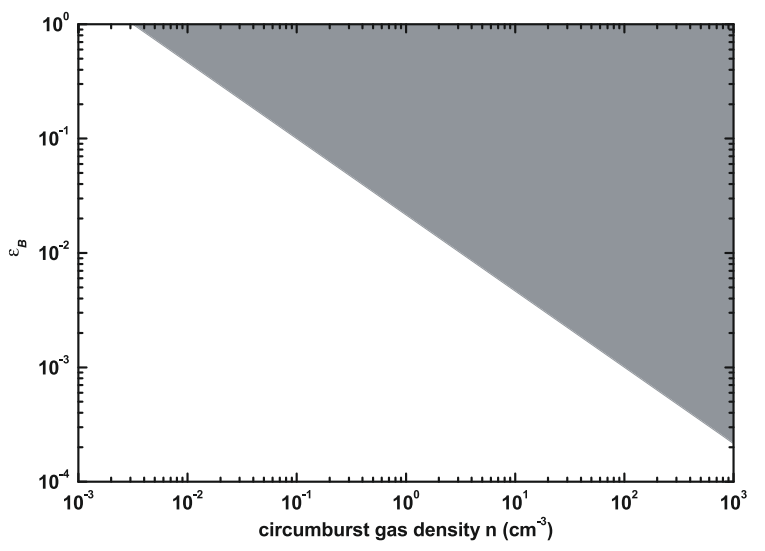

Fig. 12. Constraints on the parameter space of the fractional energy carried by the magnetic field, $\varepsilon_{B}$, and the gas density (in units of $\mathrm{cm}^{-3}$ ) in the circumburst medium into which the fireball was expanding. It was assumed here that at 0.43 days the cooling frequency was at frequencies less than $4.5 \times 10^{14} \mathrm{~Hz}\left(R_{\mathrm{C}}\right.$ band). The allowed region is represented by the grey area.

where $n_{0}=n / 1 \mathrm{~cm}^{-3}, \varepsilon_{B,-1}=\varepsilon_{B} / 0.1$, and $t_{2}=t / 100 \mathrm{~s}$. Using $v_{\mathrm{c}}<4.5 \times 10^{14} \mathrm{~Hz}$ at $t=0.43$ days with $E_{52}=2.5$ (Butler et al. 2007) and $z=3.773$ we can constrain the product $\varepsilon_{B,-1}^{-3 / 2} n_{0}^{-1}$ (Fig. 12). Since $\varepsilon_{B}<1$, it must be $n \gtrsim 0.005 \mathrm{~cm}^{-3}$, a reasonable result. On the other hand, the low deduced hydrogen column density along the line of sight in the GRB host (Sects. 3.1, 3.2) might indicate a relatively low circumburst gas density. If we require $n<100 \mathrm{~cm}^{-3}$ then $\varepsilon_{B}>0.001$, which is also a reasonable constraint.

In the following we consider the break at 0.27 days as a classical jet break and we use the results of the joint fit to discuss the energetics of the afterglow.

\subsection{Energetics}

\subsubsection{The burst}

We follow the standard approach to calculate the jet half-opening angle for an ISM environment (cf. Sari et al. 1999),

$\Theta_{\text {jet }}^{\mathrm{ISM}}=\frac{1}{6}\left(\frac{t_{\mathrm{b}}}{1+z}\right)^{3 / 8}\left(\frac{n_{0} \eta_{\gamma}}{E_{52}}\right)^{1 / 8} \mathrm{rad}$.

Here, $E_{52}$ is the isotropic equivalent energy of the prompt emission in units of $10^{52} \mathrm{erg}, n_{0}$ is the density of the ambient medium in $\mathrm{cm}^{-3}, \eta_{\gamma}$ is the efficiency of the shock in converting the energy of the ejecta into gamma radiation, and $t_{\mathrm{b}}$ is the break time in days. We set $n_{0}=1 \mathrm{~cm}^{-3}$ and $\eta_{\gamma}=0.2$. Assuming the observed break time at $t_{\mathrm{b}}=0.27 \pm 0.02$ days, as follows from the joint fit, with $E_{52}=2.5_{-0.6}^{+3.1}$ (Butler et al. 2007) we get $\Theta_{\text {jet }}^{\mathrm{ISM}}=2.37_{-0.10}^{+0.37}$ degrees. This can be compared with a mean value of the half-opening angle of the pre-Swift era GRBs of 4 degrees (Zeh et al. 2006) with a width of 0.13 dex. So, the jet of GRB 060605 was narrowly beamed but the value found is not extraordinary. Changing $\eta_{\gamma}$ to 1.0 does not increase $\Theta_{\text {jet }}^{\text {ISM }}$ in a notable manner due to the weak dependence of it on $\eta_{\gamma}$. On the other hand, as already stated before (see Eq. (1)), a very high gas density seems to be unlikely given that we do not see so much hydrogen at the redshift of the burst in the X-ray spectrum and in the optical spectrum as well. Assuming the above numbers, the corresponding beaming-corrected energy release in the gamma-ray band is $E_{\gamma}^{\text {corr }}=2.14_{-0.52}^{+2.76} \times 10^{49} \mathrm{erg}$. This is among 
the smallest $E_{\gamma}^{\text {corr }}$ found so far but, again, not exceptionally small (cf. Zeh et al. 2006; Racusin et al. 2008). The small $\Theta_{\text {jet }}^{\text {ISM }}$ and $E_{\gamma}^{\text {corr }}$ match into the picture (Racusin et al. 2008), according to which Swift GRBs have on average smaller jet opening angles and lower collimated $\gamma$-ray energies than pre-Swift bursts (which were on average at lower redshift).

As outlined by Panaitescu \& Kumar (2000), assuming that the observed peak in the optical light curve signals the fireball deceleration timescale (which is $t_{\text {peak }} /(1+z)$ ), one can calculate the initial Lorentz factor, $\Gamma_{0}$, of the outflow. Following Sari et al. (1999; Eq. (2)), in the afterglow deceleration phase the time evolution of the Lorentz factor is given by

$\Gamma(t)=6\left(\frac{E_{52}}{\eta_{\gamma} n_{0}}\right)^{1 / 8}\left(\frac{t}{1+z}\right)^{-3 / 8}$.

Setting $E_{52}=2.5$ (Butler et al. 2007), $\eta_{\gamma}=0.2$, and $n_{0}=1 \mathrm{~cm}^{-3}$, it follows $\Gamma\left(t=t_{\text {peak }}\right)=116 \pm 15$, and hence $\Gamma_{0}=232 \pm 15$. Following the procedure outlined in Molinari et al. (2007) leads to $\Gamma_{0}=366 \pm 23$. These values are comparable to those of other bursts (cf. Molinari et al.).

\subsubsection{The X-ray afterglow}

The luminosity of the afterglow is (e.g., Nousek et al. 2006)

$L_{\mathrm{X}}\left(t_{\text {host }}\right)=4 \pi d_{\mathrm{L}}^{2}(1+z)^{\beta-1} F_{\mathrm{X}}\left(t_{\mathrm{obs}}\right)$,

where $F_{\mathrm{X}}\left(t_{\mathrm{obs}}\right)$ is the observed time-dependent flux in the X-ray band and $d_{\mathrm{L}}$ is the luminosity distance. Using $z=3.773$ and assuming $\beta=1.06$ (Sect. 3.2) even at very early times, we get for the time evolution of the X-ray luminosity of the afterglow in the $0.3-10 \mathrm{keV}$ energy band (in units of erg s $\mathrm{s}^{-1}$ )

$L_{\mathrm{X}}(t)=7.0 \times 10^{48}\left(t / t_{1}\right)^{-\alpha}, \alpha=0.32 ; t_{1} \leq t \leq t_{2}$,

$L_{\mathrm{X}}(t)=2.0 \times 10^{48}\left(t / t_{2}\right)^{-\alpha}, \alpha=1.54 ; t_{2} \leq t \leq t_{3}$,

$L_{\mathrm{X}}(t)=2.6 \times 10^{47}\left(t / t_{3}\right)^{-\alpha}, \alpha=2.56 ; t \geq t_{3}$,

where $t=t_{\text {host }}$ is measured in the GRB host frame and all the break times $t_{1}, t_{2}$ and $t_{3}$ are also given in the host frame. Note that for reasons of simplicity we have replaced here the complicated light curve fit of the afterglow with a multiple broken power-law by three single power-law decays in between the deduced break times, with $t_{1}=210 \mathrm{~s} /(1+z)=44 \mathrm{~s}$ (Sect. 3.2), $t_{2}=0.072$ days $/(1+z)=0.015$ days (Table 5), and $t_{3}=0.27$ days $/(1+z)=0.056$ days (Table 5$)$. Since the breaks in the light curve are quite sharp, this is surely justified. The behaviour at $t<44 \mathrm{~s}$ (host frame) we have excluded here since it might not represent afterglow light. Based on these numbers we find that the isotropic energy release of the afterglow in the X-ray band was $4.1 \times 10^{52}$ erg between 0.0005 and 0.015 days (which is $16 \%$ of $E_{\gamma}$; Butler et al. 2007), $2.5 \times 10^{51}$ erg between 0.015 and 0.056 days $\left(0.10 E_{\gamma}\right)$, and $8.1 \times 10^{50} \mathrm{erg}$ thereafter $\left(0.03 E_{\gamma}\right)$, assuming a constant decay.

\subsubsection{The optical afterglow}

Similar to the "Bronze Sample" of Kann et al. (2007), we can assume that the $R_{\mathrm{C}}$-band afterglow of GRB 060605 is not affected by host galaxy extinction (which seems to be low at high redshifts anyway, Kann et al. 2007). This assumption is also supported by the observed SED of the afterglow at 0.43 days (Fig. 10). Furthermore, if the cooling break $v_{\mathrm{c}}$ lies at wavelengths longer than the optical ones then $\beta_{\mathrm{opt}}=\beta_{\mathrm{X}}$. Therefore,

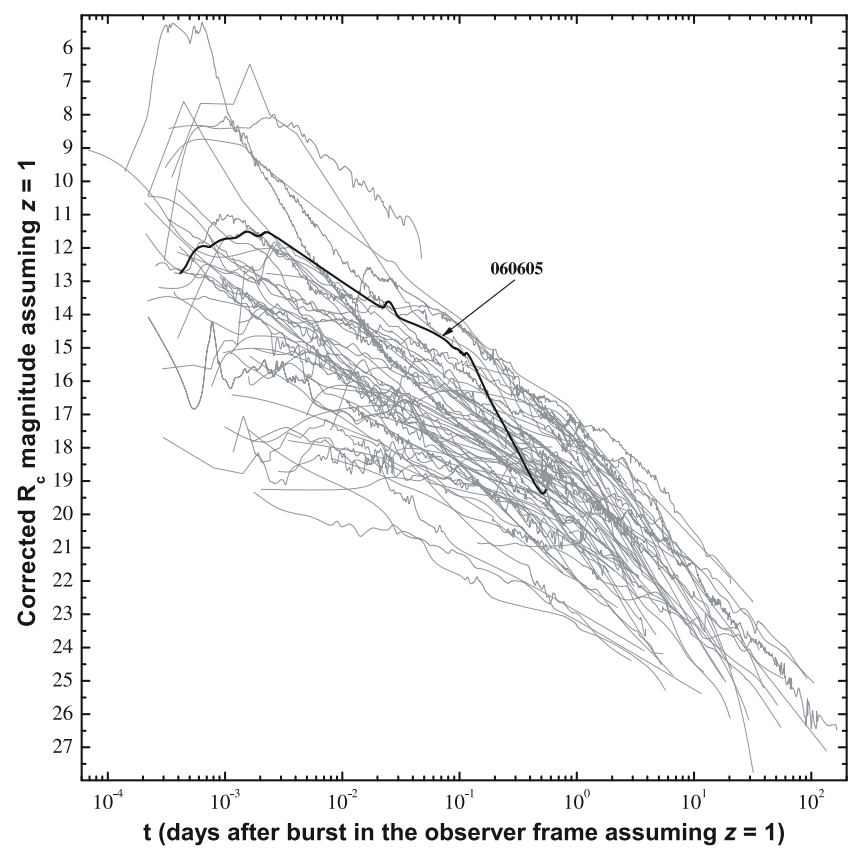

Fig. 13. The observed $R_{\mathrm{C}}$-band light curve of the afterglow of GRB 060605 compared to the ensemble of optical afterglows known so far after shifting all light curves to a common redshift of $z=1$. All data are corrected for Galactic extinction. See Kann et al. (2006) for the method and Kann et al. (2007) for more details on other bursts.

assuming $A_{V}$ (host) $=0, \beta_{\mathrm{opt}}=1.06$, and using the method presented in Kann et al. (2006), we are able to derive a lower limit on the magnitude shift $d R_{\mathrm{c}} \geq 3.61 \mathrm{mag}$. This shift (see Kann et al. 2006, for more details) describes the magnitude change that appears when the afterglow light curve is corrected for extinction (which we are unable to do here, therefore we derive only a lower limit) and shifted to $z=1$ (which also implies a temporal shift). Comparing the afterglow with the sample presented in Kann et al. (2007), we find that it is among the brightest afterglows at early times, comparable to the afterglow of GRB 050820A (Fig. 13). At $43 \mathrm{~s}$ in the rest-frame $(z=1$ assumed), it has $R_{\mathrm{C}} \leq 11.83 \pm 0.15$, which places it among the tight clustering found by Kann et al. (2007), although the afterglow is still rising. To derive a magnitude at one day after the GRB (if at $z=1$ ), we need to extrapolate the late steep decay. We find $R_{\mathrm{C}} \leq 20.9 \pm 0.2\left(M_{B} \leq-22.0 \pm 0.2\right.$; assuming no host extinction), which is relatively faint. At a similar redshift, only the afterglow of GRB 050502A was fainter (Kann et al. 2007).

\subsection{The host galaxy}

To search for the host galaxy of GRB 060605 and the potential foreground absorber detected in our optical spectrum (Fig. 3) we used VLT/FORS2 imaging data of the field obtained under the ESO Large Programme 177.A-0591 (PI: Jens Hjorth). Based on images obtained with the $3.5 \mathrm{~m}$ Italian Telescopio Nationale Galileo on La Palma (Malesani et al. 2006; Deng et al., in preparation) we were able to derive an improved astrometric position of the optical transient. Its refined position is RA $(\mathrm{J} 2000)=21^{\mathrm{h}} 28^{\mathrm{m}} 37^{\mathrm{s}} 314$ and Dec $=-06^{\circ} 3^{\prime} 30^{\prime}{ }^{\prime} 88$. On the deep VLT image the afterglow can be positioned with an accuracy of 0.1 arcsec. At this position a very faint extended source is visible (Fig. 14). Using the average zeropoint of FORS2 $R_{\mathrm{C}}$-band images in the time period from July to September 2007 $(28.404 \pm 0.037)$, as it is provided on ESO's web pages, we 


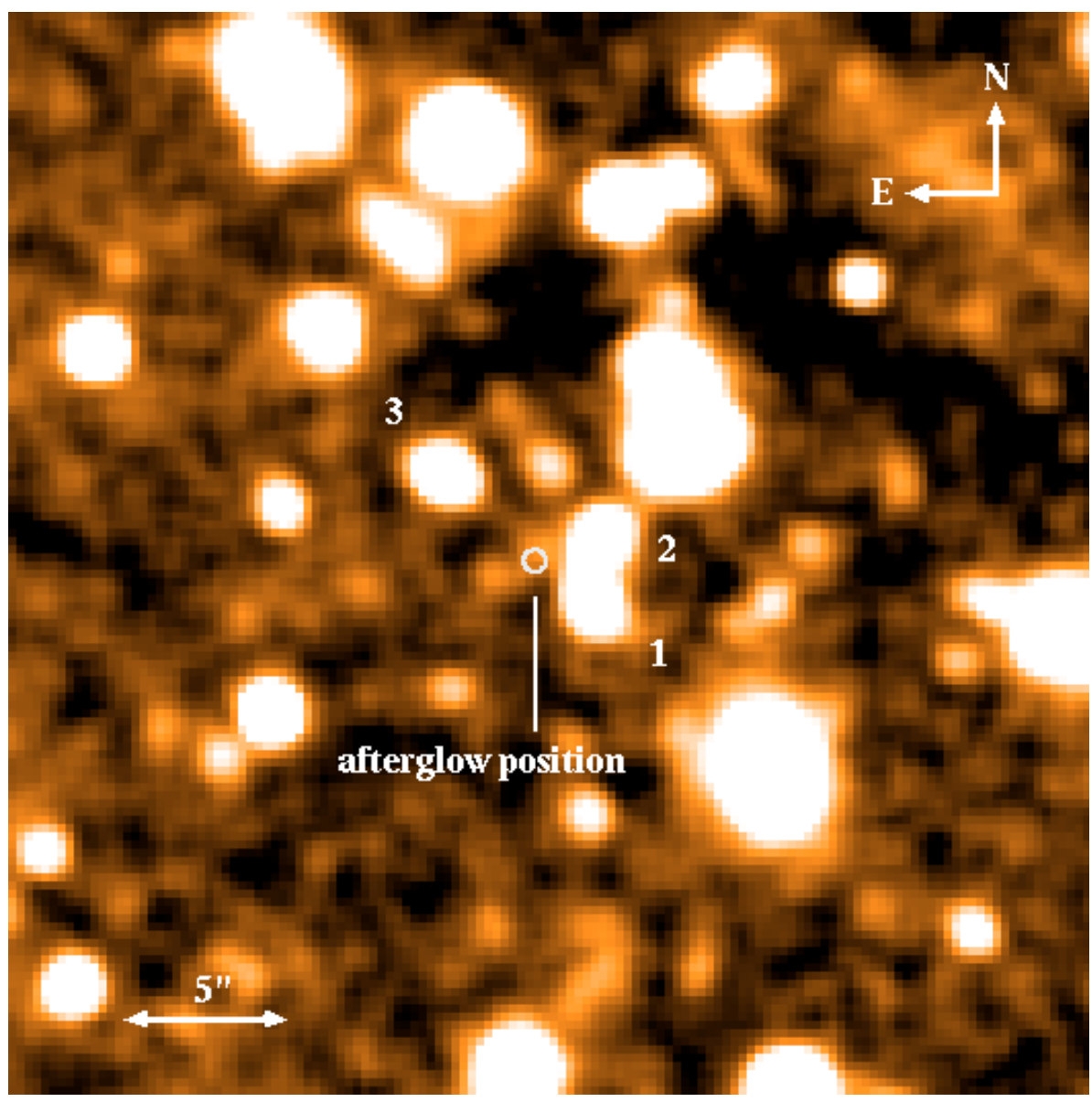

Fig. 14. The stellar field around the position of the afterglow of GRB 060605. The $R_{\mathrm{C}}$-band image is the average of $8 \times 500$ s exposure time each, taken on 14 Sep. 2006 and 18 Jun. 2007 at VLT/FORS2, when the afterglow had faded away. The position of the optical transient is indicated. The faint extended source underlying the position of the optical transient is the suspected GRB host galaxy at $z=3.773$. The three brightest galaxies next to it are indicated with numbers. derive a magnitude of $R_{\mathrm{C}}=26.4 \pm 0.3$ for this source (aperture diameter $=10$ pixels). If this is the host galaxy, it implies that at its peak time the optical afterglow of GRB 060605 was approximately 11 mag brighter in $R_{\mathrm{C}}$ than its host. We caution, however, that the detection is weak and we cannot claim that this object is the host of GRB 0606005 as we have no information about its redshift. On the other hand, its position underlying the optical transient and its faint magnitude make a potential physical association with GRB 060605 a reasonable assumption.

One can also speculate if any of the other three bright, extended sources seen near the afterglow position on the VLT image could be the host galaxy of GRB 060605. In Fig. 14 these three galaxies are indicated with the numbers 1, 2, and 3 . However, there are two arguments against this hypothesis. First, these galaxies have $R_{\mathrm{C}} \sim 24.5$. For an assumed redshift of $z=3.7$ this would place all of them at the very bright end of the Schechter luminosity function (cf. Lin et al. 1996). Second, the angular distance of the optical transient from the centres of these galaxies is $2{ }^{\prime \prime} 12,22^{\prime \prime} 27$, and $33^{\prime \prime} 94$, respectively. For the considered world model at a redshift of $z=3.773$ an angular distance of 1 arcsec corresponds to a projected distance of $7.26 \mathrm{kpc}$. The projected distance of the optical transient from the three galaxies is then $15.4,16.5$ and $28.6 \mathrm{kpc}$, respectively. Compared to the offset distribution of GRBs with respect to their host galaxies (in the pre-Swift era; Bloom et al. 2002) these large distances make it unlikely that one of these galaxies is the host. Finally, using basically the same arguments it is unlikely that one of them is the foreground absorber seen in the optical spectrum of the optical transient at $z=3.709$ (Sect. 3.1). On the other hand, the foregroud absorber could be the faint object to the south-east of the possible host galaxy.

\section{Summary}

We have reduced and analysed XRT and UVOT data from Swift and integral field unit spectra of the afterglow of GRB 060605. In addition, VLT images were obtained to search for the GRB host galaxy. We find: (1) the afterglow spectrum reveals two absorption line systems at redshifts 3.773 and 3.709 . We identify the former with the redshift of the burst. (2) The deduced measured H I column density for the host galaxy is in between $N_{\mathrm{HI}}=10^{18.5}$ and $10^{19.3} \mathrm{~cm}^{-2}$. It is one of the lowest ever detected in a GRB afterglow. (3) From the observed time evolution of the X-ray/optical SED, pointing to a decrease of $v_{\mathrm{c}}$ with time, we conclude that the afterglow propagated into an ISM environment. The cooling frequency was below the $R$ band $\left(v_{\mathrm{c}}<v_{R}\right)$ after the jet break time. The initial Lorentz factor of the fireball was about 250. (4) Our analysis of the X-ray light curve suggests that it followed the canonical X-ray light curve shape (Nousek et al. 2006; Zhang et al. 2006). A comparison of the $\mathrm{X}$-ray and the optical afterglow light curves reveals an achromatic evolution at late times. (5) The observed jet break time is at 0.27 days. This early jet break time (in the GRB host frame at about $4900 \mathrm{~s}$ after the burst) is the most remarkable property of GRB 060605. (6) The early observed jet break translates into a relatively small beaming angle of 2.4 degrees and hence a relatively small beaming-corrected energy release in the gammaray band of about $2.1 \times 10^{49} \mathrm{erg}$. These values are not exceptionally low, however (cf. Zeh et al. 2006; Racusin et al. 2008). In the X-ray band the afterglow released an energy that is of comparable amount. In the optical, at early times, the afterglow was among the most luminous ever detected. (7) A faint $\left(R_{\mathrm{C}}=26.4 \pm 0.3\right)$, extended source seen on VLT images at the 
position of the afterglow might be the GRB host galaxy or the foreground absorber seen in the optical spectrum.

The detailed study of this burst was triggered by our goal to use integral field units to perform rapid follow-up observations of arcsec-sized Swift X-ray error circles. While in this particular case our observations were performed only some hours after the event, we could obtain useful spectra. One can imagine that a much faster response with an integral field unit, immediately after the announcement of an arcsec-sized Swift X-ray error circle, can provide early spectral information on bursts.

Acknowledgements. P.F., S.K. and D.A.K., acknowledge financial support by DFG grant K1 766/13-2 and by the German Academic Exchange Service (DAAD) under grant No. D/05/54048. S.K. and S.S. thank Kim Page (Leicester) for useful discussions. The research activities of J.G. are supported by the Spanish Ministry of Science and Education through projects AYA2004-01515 and ESP2005-07714-C03-03. Swift is supported at PSU by NASA contract NAS5-00136. The Dark Cosmology Centre is funded by the Danish National Research Foundation. We thank the Calar Alto and the ESO staff for excellent support and the referee for a very careful reading of the manuscript and very helpful remarks.

\section{References}

Barthelmy, S. D., Barbier, L. M., Cummings, J. R., et al. 2005, Space Sci. Rev., 120,143

Becker, T. 2002, Ph.D. Thesis, University of Potsdam

Bessell, M. S. 1979, PASP, 95, 589

Beuermann, K., Hessman, F. V., Reinsch, K., et al. 1999, A\&A, 352, L26

Blackburn, J. K. 1995, FTOOLS: A FITS Data Processing and Analysis Software Package, in: Astronomical Data Analysis Software and Systems IV, ed. R. A. Shaw, H. E. Payne, \& J. J. E. Hayes (San Francisco: ASP), ASP Conf. Ser., 77,367

Bloom, J. S., Kulkarni, S. R., \& Djorgovski, S. G. 2002, AJ, 123, 111

Blustin, A. J., \& Page, M. J. 2006, GCN Circular, 5228

Burrows, D. N., Hill, J. E., Nousek, J. A., et al. 2005, Space Sci. Rev., 120, 165

Butler, N. R., Kocevski, D., Bloom, J. S., \& Curtis, J. L. 2007, ApJ, 671, 656

Cavallo, G., \& Rees, M. J. 1978, MNRAS, 183, 359

Chen, H.-W., Prochaska, J. X., Ramirez-Ruiz, E., et al. 2007a, ApJ, 663, 420

Chen, H.-W., Prochaska, J. X., \& Gnedin, N. 2007b, ApJ, 667, L125

Chevalier, R. A., \& Li, Z-Y. 2000, ApJ, 536, 195

Dickey, J. M., \& Lockman, F. J. 1990, ARA\&A, 28, 215

Evans, P. A., Beardmore, A. P., Page, K. L., et al. 2007, A\&A, 469, 379

Fiore, F., D'Elia, V., Lazzati, D., et al. 2005, ApJ, 624, 853

Fynbo, J. U. P., Gorosabel, J., Dall, T. H., et al. 2001, A\&A, 373, 796

Gehrels, N., Chincarini, G., Giommi, P., et al. 2004, ApJ, 611, 1005

Godet, O., Page, K. L., Rol, E., Beardmore, A. P., \& Page, M. J. 2006, GCN Circular, 5227

Granot, J., Piran, T., \& Sari, R. 2000, ApJ, 534, L163

Greiner, J., Klose, S., Salvato, M., et al. 2003, ApJ, 599, 1223

Grupe, D., Nousek, J. A., vanden Berk, D. E., et al. 2007a, AJ, 133, 2216

Grupe, D., Gronwall, C., Wang, X.-Y., et al. 2007b, ApJ, 662, 443

Jakobsson, P., Fynbo, J. P. U., Ledoux, C., et al. 2006, A\&A, 460, L13

Jin, Z. P., \& Fan, Y. Z. 2007, MNRAS, 378, 1043

Kalberla, P. M. W., Burton, W. B., Hartmann, D., et al. 2005, A\&A, 440, 775

Kann, D. A., Klose, S., \& Zeh, A. 2006, ApJ, 641, 993

Kann, D. A., Klose, S., Zhang, B., et al. 2007, ApJ, submitted [arXiv:0712.2186]
Karska, A., \& Garnavich, P. 2006, GCN Circular, 5260

Kelz, A., Verheijen, M. A. W., Roth, M. M., et al. 2006, PASP, 118, 129

Khamitov, I., Saygac, A. T., Aslan, Z., et al. 2006a, GCN Circular, 5224

Khamitov, I., Saygac, A. T., Aslan, Z., et al. 2006b, GCN Circular, 5235

Klose, S., Greiner, J., Rau, A., et al. 2004, AJ, 128, 1942

Liang, E.-W., Zhang, B.-B., \& Zhang, B. 2007, ApJ, 670, 565

Liang, E.-W., Racusin, J. L., Zhang, B., Zhang, B.-B., \& Burrows, D. N. 2008, ApJ, 675, 528

Lin, H., Kirshner, R. P., Shectman, S., et al. 1996, ApJ, 464, 60

Malesani, D., Fiore, F., Masetti, N., Pedani, M., \& Mainella, G. 2006, GCN Circ., 5225

Mészáros, P. 2006, Rep. Progr. Phys., 69, 2259

Mirabal, N., Halpern, J. P., Chornock, R., et al. 2003, ApJ, 595, 935

Molinari, E., Vergani, S. D., Malesani, D., et al. 2007, A\&A, 469, L13

Nardini, M., Ghisellini, G., \& Ghirlanda, G. 2008, MNRAS, 386, L87

Nousek, J. A., Kouveliotou, C., Grupe, D., et al. 2006, ApJ, 642, 389

O’Brien, P. T., Willingale, R., Osborne, J., et al. 2006, ApJ, 647, 1213

Oke, J. B. 1990, AJ, 99, 1621

Page, M. J., Blustin, A. J., Brown, P. J., et al. 2006, GCN Circular, 5221

Panaitescu, A. 2007, MNRAS, 380, 374

Panaitescu, A., \& Kumar, P. 2000, ApJ, 543, 66

Peterson, B., \& Schmidt, B. 2006, GCN Circular, 5223

Piran, T. 2005, Rev. Mod. Phys., 76, 1143

Poole, T. S., Breeveld, A. A., Page, M. J., et al. 2008, MNRAS, 383, 627

Racusin, J. L., Liang, E. W., Burrows, D. N., et al. 2008, ApJ, submitted [arXiv: 0812.4780]

Rees, M. J., \& Mészáros, P. 1992, MNRAS, 258, 41

Roming, P. W. A., Kennedy, T. E., Mason, K. O., et al. 2005, Space Sci. Rev., 120,95

Roming, P. W. A., Schady, P., Fox, D. B., et al. 2006, ApJ, 652, 1416

Roth, M. M., Kelz, A., Fechner, T., et al. 2005, PASP, 117, 620

Rykoff, E. S., \& Schaefer, B. E. 2006, GCN Circular, 5220

Sánchez, S. F. 2004, Astron. Nachr., 325, 167

Sánchez, S. F. 2006, Astron. Nachr., 327, 850

Sánchez, S. F., \& Cardiel, N., 2005, Calar Alto Newsletter, 10

Sari, R. 1997, ApJ, 489, L37

Sari, R., Piran, T., \& Narayan, R. 1998, ApJ, 497, L17

Sari, R., Piran, T., \& Halpern, J. P. 1999, ApJ, 519, L17

Sato, G., Barbier, L., Barthelmy, S., et al. 2006, GCN Circular, 5231

Savaglio, S. 2006, New J. Phys., 8, 195

Savaglio, S., \& Fall, S. M. 2004, ApJ, 614, 293

Savaglio, S., Fiore, F., Israel, G., et al. 2002, GCN Circular, 1633

Schaefer, B. E., Gerardy, C. L., Höflich, P., et al. 2003, ApJ, 588, 387

Schaefer, B. E., Rykoff, E. S., Smith, D. A., \& Quimby, R. 2006, GCN Circular, 5222

Schlegel, D. J., Finkbeiner, D. P., \& Davis, M. 1998, ApJ, 500, 525

Shapley, A. E., Steidel, C. C., Adelberger, K. L., et al. 2001, ApJ, 562, 95

Sharapov, D., Augusteijn, T., \& Pozanenko, A. 2006, GCN Circular, 5263

Shin, M.-S., Berger, E., Penprase, B. E., et al. 2006, ApJ, submitted [arXiv:astro-ph/0608327]

Spergel, D. N., Verde, L., Peiris, H. V., et al. 2003, ApJS, 148, 175

Starling, R. L. C., van der Horst, A. J., Rol, E., et al. 2008, ApJ, 672, 433

Still, M., Kniazev, A., Romero-Colmenero, E., et al. 2006, GCN Circular, 5226

Vaughan, S., Goad, M. R., Beardmore, A. P., et al. 2006, ApJ, 638, 920

Verheijen, M. A. W., Bershady, M. A., Andersen, D. R., et al. 2004, Astron. Nachr., 325, 151

Watson, D., Hjorth, J., Fynbo, J. P. U., et al. 2007, ApJ, 660, L101

Zeh, A., Klose, S., \& Kann, D. A. 2006, ApJ, 637, 889

Zhai, M., Qiu, Y. L., Wei, J. Y., Hu, J. Y., Deng, J. S., \& Zheng, W. K. 2006, GCN Circ., 5230

Zhang, B., \& Mészáros, P. 2004, Int. J. Mod. Phys. A, 19, 2385

Zhang, B., Fan, Y. Z., Dyks, J., et al. 2006, ApJ, 642, 354 